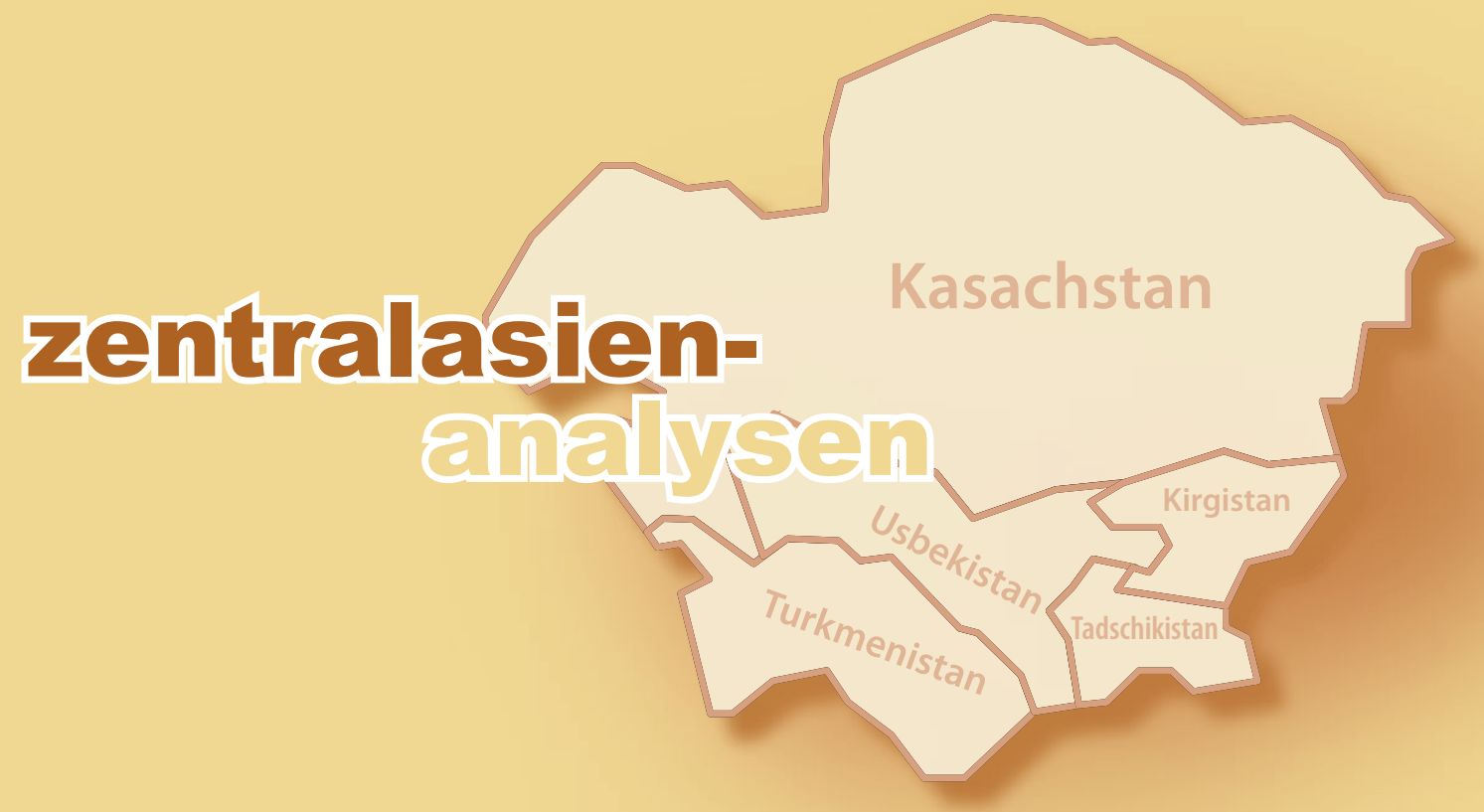

www.laender-analysen.de/zentralasien

\title{
ISLAM IN ZENTRALASIEN
}

\section{ANALYSE}

Der politische Islam in Zentralasien - Gegner oder demokratischer Partner?

Von Arne C. Seifert, Berlin

- TABELLEN UND GRAFIKEN ZUM TEXT

Zentralasien im Human Development Index

Ausgewählte sozioökonomische Grunddaten

Religiöse Orientierung in Tadschikistan

\section{CHRONIK}

Vom 19. Mai bis zum 22. Juni 2012 


\title{
Der politische Islam in Zentralasien - Gegner oder demokratischer Partner?
}

\author{
Von Arne C. Seifert, Berlin
}

\section{Zusammenfassung}

Der politische Islam ist im heutigen Zentralasien eine feste Größe, deren Bedeutung unter den gegenwärtigen politischen und sozioökonomischen Bedingungen eher wachsen als verschwinden wird. Darauf müssen sich nicht nur die herrschenden Eliten einstellen, sondern auch westliche Politik. Politischer Islam ist keine uniforme, westlichen Vorstellungen grundsätzlich ablehnend gegenüberstehende Erscheinung. Es gibt auch moderate Kräfte, die islamische Werte und nationale Interessen verbinden, sie treten aber bislang wenig in Erscheinung. Noch hat westliche Politik die Chance, mit ihnen zusammenzuarbeiten und so auf eine moderate Ausrichtung des politischen Islam in den zentralasiatischen Staaten hinzuwirken. Aber die Zeit drängt.

I $\mathrm{m}$ eurasischen Raum ist der politische Islam nicht nur eine religiöse, sondern auch eine gesellschaftspolitische Größe, die aus dem politischen Leben nicht ausgegrenzt werden kann. Daher ist die grundsätzliche Frage nach dem Verhältnis zum politischen Islam und seinen Trägern zu klären. Ein produktiver Ausgangspunkt muss der Vielfalt der Interessen, zivilisatorischen Prägungen und Religionen sowie nationalen und ethnischen Belangen Rechnung tragen.

Der für Europa wohl naheliegendste Grund, sich für Vertrauensbildung mit dem politischen Islam in Zentralasien einzusetzen, ist das absehbare Ende der militärischen Präsenz des Westens in Afghanistan. Zwar stellt man sich in den zentralasiatischen Staaten, aber auch in Russland und China die bange Frage, ob die Taliban den islamistischen Untergrund in Zentralasien mobilisieren können; die mindestens ebenso wichtige Anschlussfrage, ob man nicht wenigstens die zentralasiatische Sektion des politischen Islam gegen externe Manipulation »immunisieren" könnte, wird hingegen (noch) nicht gestellt. Initiativen aus Europa zur Einleitung eines Prozesses der Vertrauensbildung könnten hier eine wichtige Rolle spielen.

Zumindest in Kirgistan, Tadschikistan und Usbekistan besteht ein beträchtliches Konfliktpotenzial, einschließlich eines extremistisch-islamistischen Untergrunds. Zwar weiß niemand genau, wie stark er ist, doch kann man davon ausgehen, dass der extremistische Islamismus angesichts des hohen sozioökonomischen und politischen Problemstaus in diesen Ländern auf ein beträchtliches Mobilisierungspotenzial in der muslimischen Bevölkerungsmehrheit zurückgreifen kann. Dass dieser Untergrund auf eine gewaltsame Veränderung der säkularen politischen Systeme zielt, ist hinlänglich bekannt. Als die Taliban von 1996/97 bis 2001 in Afghanistan an der Macht waren, beeinflussten sie die Regelung des tadschikischen Bürgerkriegs (19921997) und boten der Islamischen Bewegung Usbekistans (IMU) militärisches Hinterland für ihr Vordringen nach Usbekistan (1999, 2000). Seitdem erweisen sie logistische Hilfestellung und könnten als Druckmittel gegen den Westen zu einer dschihadistischen Mobilisierung in Zentralasien beitragen.

Brisanz gewinnt die Situation durch das Fehlen eines wirksamen moderaten islamischen Gegengewichts zu den extremistischen Orientierungen. Ein solches Gegengewicht müsste von reformerischen, auf die Konsolidierung ihrer jungen Staaten gerichteten Kräften gebildet werden. Dass es solche Kräfte gibt, konnte man z. B. bei Veranstaltungen und vertrauensbildenden Dialogen des Zentrums für OSZE-Forschung (CORE) in Kasachstan, Kirgistan und Tadschikistan beobachten. Sie gehören meist der jüngeren Generation an, sind gut gebildet und zeichnen sich durch eine Symbiose des Bekenntnisses $\mathrm{zu}$ islamischen Werten und nationalen Interessen aus. Dass sie politisch nicht ins Gewicht fallen, liegt in erster Linie daran, dass ihnen die säkularen Herrschaftsträger den Weg zur Profilierung als legale politische Akteure versperren. Lediglich in Tadschikistan vermochte die "Partei der islamischen Wiedergeburt" (PIWT), durch die Vereinbarungen zur Beendigung des Bürgerkriegs einen legalen Status zu erringen; sie ist im Parlament als Oppositionspartei vertreten. Aber auch die PIWT sieht sich weiterhin mit erheblichem Widerstand seitens der Staatsmacht konfrontiert. Da der extremistische Untergrund ohne das Entstehen genuiner alternativer islamischer Bewegungen jedoch nicht aus der Welt zu schaffen ist, gibt es zu deren Legalisierung als politische Akteure und ihrer Einbeziehung in den nationalen wie eurasischen Dialog keine Alternative.

\section{Wachsender Einfluss des Islam in der Bevölkerung}

Im Jahr 2000 bekannten sich $63 \%$ der kasachstanischen, $82 \%$ der usbekischen und $79 \%$ der tadschikischen Bevölkerung zu dem Prinzip »es gibt keinen Gott außer Allah". Man kann davon ausgehen, dass die Anhängerschaft des Islam seitdem noch beträchtlich gewachsen ist. In Tadschikistan verstehen sich nach soziologischen Untersuchungen inzwischen $94,8 \%$ der 
Bevölkerung als Muslime und die Bedeutung der Religion ist als Werteorientierung außerordentlich gestiegen. Der Islam kommt in der tadschikischen Gesellschaft vor allem als moralisches Wertesystem zur Geltung, als Überzeugung und Anschauung, die den Sinn des Individuums bestimmen. $96 \%$ der Tadschiken lassen sich bei ihren Entscheidungen mehr oder weniger von religiösen Erwägungen leiten, 73,7 \% besuchen eine Moschee relativ regelmäßig, $50 \%$ beten täglich fünf Mal, 76,2\% fasten während des Ramadan.

Die Islamisierung der Bevölkerung drückt sich nicht nur in Zahlen aus, sondern auch in einer zunehmenden Bereitschaft, islamische Verhaltensvorschriften zu befolgen. So nehmen Geschäftsinhaber in Tadschikistan und Kirgistan Umsatzverluste in Kauf, weil sie den Verkauf alkoholischer Getränke einstellen. In einigen Gebieten der kirgisischen Hauptstadt Bischkek achten jugendliche Gläubige auf die Einhaltung der Regeln islamischer Schlachtung. Hochzeiten nach islamischem Ritus, das Tragen des islamischen Kopftuches und die Einhaltung islamischer Feiertage nehmen überall zu. Nationale Unternehmer spenden an islamische Einrichtungen, die Praxis islamischen Bankings verbreitet sich zunehmend. Auch beschränkt sich der wachsende Einfluss des Islam nicht mehr nur auf sozial schlechter gestellte Bevölkerungsschichten, sondern breitet sich auch in studentischen Kreisen, der Intelligenz sowie in der kleinen und mittleren Unternehmerschaft aus. Vor allem aber erfasst er die ländliche Bevölkerungsmehrheit.

In Kirgistan, das bisher als traditionell weniger religiös galt, neigt heute die Masse der Bevölkerung zwischen 30 und 64 Jahren zur Identifikation mit einem national orientierten Islam. Und selbst in Kasachstan, das für weniger aufnahmebereit für den Islam gehalten wurde, verweisen soziologische Untersuchungen auf eine steigende Religiosität der Bevölkerung. Kasachstanische Experten befürchten, dass eine wachsende Anzahl weltanschaulich noch ungefestigter Jugendlicher in den Wirkungsbereich von Predigern eines fundamentalistischen Islam gerät, was bereits in absehbarer Zeit dazu führen könnte, dass sie sich ökonomisch und politisch der heutigen Gesellschaft Kasachstans entfremden und es zur Formierung einer neuen Generation religiöser Fanatiker kommt. Insgesamt nimmt die Anziehungskraft des Islam auf die Bevölkerung deutlich zu und prägt deren weltanschauliche und teils auch schon sozialpolitische Vorstellungswelt. Nicht weniger wichtig ist die Kehrseite dieses Prozesses: Je breiter sich der Islam erneut "vergesellschaftet«, desto dünner wird die säkulare Bodenschicht, die in der sowjetischen Periode von außen aufgetragen wurde und in der sich das Staatsverständnis der herrschenden Eliten sowie beträchtlicher Teile der Intelligenz begründet. Anders gesagt: Die sozialen Grund- lagen des Säkularismus werden ausgedünnt, was seine gesellschaftlichen Legitimationsgrundlagen schwächt.

\section{Transformation und Islamisierung}

Der gegenwärtige Aufstieg des Islam in Zentralasien ist in erster Linie nicht das Ergebnis der Agitation von Missionaren. Vielmehr ist er Resultat einer Reihe ineinandergreifender ökonomischer, politischer und historischer Faktoren. In diesem Zusammenhang gilt es sich zu vergegenwärtigen, dass der Islam auch während der sowjetischen Periode als System religiöser moralischer Grundsätze und täglicher ritueller Übungen weiterlebte, das durchaus auch als Alternative zum bestehenden System gesehen wurde. Das bedeutet, dass die soziale Basis des Islam nie vollends ausgetrocknet war. Dass seine "Reanimation " jedoch in einem historisch beispiellosen Tempo von nur etwa zwanzig Jahren erfolgen konnte, ist in hohem Maße auf die Transformationsund Staatsformungsprozesse in Zentralasien zurückzuführen, die der Westen entscheidend mit beeinflusst hat. Sein Bestreben war es, alle politischen, wirtschaftlichen und gesellschaftlichen Systeme quasi in einer Frontalstrategie mit einer gleichzeitigen Transformation umzugestalten. Die sozioökonomischen Folgen dieser Schocktherapie vermochten die meisten zentralasiatischen Gesellschaften bis auf den heutigen Tag nicht zu verkraften. Die gesamte Region ist mit einer fundamentalen Verschlechterung der sozialen Lebensumstände konfrontiert. Dabei fallen nicht mehr allein die einzelnen negativen Parameter wie niedriges Pro-KopfEinkommen, große Armut, hohe Arbeitslosigkeit oder schlechte soziale Vorsorgesysteme ins Gewicht. Vielmehr geht es bereits um deren qualitativen Sprung in massenhafte soziale Ausgrenzung und Spaltung in den Gesellschaften. So gelten nach dem UNDP-Regional Human Development Report (2011), der eine Gesamtbilanz von zwanzig Jahren Transformation für Osteuropa und Zentralasien zieht, gegenwärtig sogar in Kasachstan $32 \%$ der Bevölkerung als "sozial ausgegrenzt «, in Tadschikistan sind es $72 \%$.

Angesichts dieser sozialen Problemlage gewinnt insbesondere die Hinwendung der jungen Generation zum Islam und zu islamischen Oppositionsformen an Bedeutung. Die zentralasiatischen Staaten weisen eine immer jüngere Bevölkerung auf, die jährlich um durchschnittlich 1,7\% wächst und in der inzwischen $30 \%$ jünger als 15 Jahre sind. Dieses Strukturproblem zeigt sich vor allem in der Jugendarbeitslosigkeit, die in den zentralasiatischen Staaten mit Ausnahme Kasachstans auf über $20 \%$ geschätzt wird. Der Anteil der 15-29jährigen an den tadschikischen Arbeitsmigranten beträgt $53 \%$. Von den Arbeitslosen in der tadschikischen Landwirtschaft sind 83,6\% unter 40 Jahre alt. Diese Befunde erinnern 
daran, dass man für eine realistische Einschätzung islamischer Politisierung immer auch die soziale Basis, wie sie sich infolge der Transformation entwickelt hat, im Blick haben muss. Das ist auch deshalb ein wichtiger Aspekt, weil er vor der Illusion schützt, dass es in absehbarer Zeit gelingen könnte, die soziale Basis der Islamisierung aus der Welt zu schaffen. Dies ist schon deshalb illusorisch, weil die Folgen jener Radikaltransformation beträchtliche Teile der Bevölkerung dazu gebracht haben, Zuflucht in traditionellen, partikularen Strukturen zu suchen, die der einfachen Bevölkerung als einzige noch ein gewisses Maß an sozialer Sicherheit, persönlicher Ehre und Würde bieten. Daher rührt auch die starke Wiederbelebung des gesamten Systems der Clans und Großfamilien in den letzten Jahrzehnten. Immanente Bestandteile dieser Strukturen sind Medressen, private Koranschulen und fragwürdige Formen islamischer Bildungsvermittlung, die sich zumeist durch ihr niedriges intellektuelles Niveau, ja sogar ihren Untergrundcharakter auszeichnen. Dort erwarten die Menschen zugleich Hizb ut-Tahrir al-Islami und Salafisten mit ihrer von den kritischen sozioökonomischen Umständen unterstützten Agitation für soziale Gerechtigkeit, gegen Gewalt und Korruption.

\section{Politisierung der Religion}

Die Hinwendung zur Religion als Reaktion auf Armut, Verelendung und soziale Perspektivlosigkeit ist ein seit Jahrhunderten bekanntes Phänomen. Dabei kommt es immer darauf an, in welchem Verhältnis das religiöse Element zu den sozialen Triebkräften und möglichen politischen Ausdrucksformen steht. Russische Experten gehen zurecht davon aus, dass die Politisierung der religiösen Sphäre in Zentralasien in erster Linie von sozialen Faktoren und der Nichtbewältigung elementarer Entwicklungsprobleme verursacht wird, vom Unwillen und der Unfähigkeit der Staatsmacht, die aus Rückständigkeit, Armut, Arbeitslosigkeit und Korruption erwachsenden Probleme zu lösen. Davon sind insbesondere Tadschikistan, Kirgistan und Usbekistan betroffen, insbesondere das Ferganatal, sowie der Süden Kasachstans. Insgesamt ist festzuhalten, dass der politische Islam die vorhandene soziale Energie bisher wenig politisch instrumentalisiert, beispielsweise nicht die Gläubigen zur Lösung der sozialen Fragen mobilisiert hat. Noch ist die Möglichkeit einer islamistischen Machtergreifung also als gering einzuschätzen. Die Hauptrichtung des Islamisierungsprozesses besteht derzeit in einer Vertiefung der Religiosität. Das bedeutet, dass soziale Fragen (neben dem Ringen um Religionsfreiheit) bei der Hinwendung zum Islam zwar eine große Rolle spielen, aber (noch) nicht als Triebkraft für die politische Mobilisierung der Gläubigen instrumentalisiert wer- den. Auch haben die Träger des politischen Islam bisher (noch) keine Offensive zur Umwandlung des säkularen Staates in einen islamischen gestartet.

Aber der Grat zwischen Religiosität und potenzieller politischer Mobilisierung kann sich als schmal und trügerisch erweisen. Ein anschauliches Beispiel dafür geben die zornigen jungen Tadschiken, Kirgisen und Usbeken, die als Arbeitsmigranten ihre Arbeitskraft für ein miserables Entgelt in Russland verkaufen müssen, um ihre Familien daheim ernähren zu können. Gemeinsam beten sie in Moskauer Moscheen. Der Zorn auf ihre Situation könnte wie ein Katalysator wirken, der die soziale Frage und den Islam zu einem explosiven Gemisch verbindet.

Auch ist in der Region unvergessen, dass die Umwandlung Tadschikistans in einen islamischen Staat im tadschikischen Bürgerkrieg ein wichtiges Motiv der islamischen Opposition war, genauso wie man deren Marginalisierung durch die säkulare Staatsmacht, nachdem die Islamisten Friedensvereinbarungen zugestimmt und ihre Waffen niedergelegt hatten, nicht vergessen hat. Dieser ausschließende Vorgang, bei dem eine säkular-islamische Koalitionsregierung sowie Vertrauensbildung nach einem Bürgerkrieg der Restauration säkularer Autokratie geopfert wurden, ist von beträchtlicher Relevanz für die äußerst strittige Frage, ob der politische Islam, auch in seiner radikalen, nicht aber dschihadistischen Form, einen Platz im Rahmen von Vertrauensbildung haben kann. Eine erste Antwort liefert die Gegenfrage nach den Risiken, die ein Ausschluss solcher Kräfte aus der Vertrauensbildung nach sich ziehen würde, ist doch davon auszugehen, dass auch islamischer Radikalismus noch für unbestimmte Zeit Inspiration großer Bevölkerungsschichten bleiben wird. Antwort bietet auch die positive Erfahrung eines integrierenden Umgangs mit radikalen Islamisten in Tadschikistan unter dem Druck der von der UN moderierten Friedensvereinbarungen, die die Einbeziehung führender Islamisten in nationale Regierungsverantwortung und deren Abfall von den dschihadistischen Positionen des Bürgerkriegs bewirkte. In dieser Periode (2000 bis etwa 2005) gab der frühere PIWT-Vorsitzende Said Abdullo Nuri die Strategie der Schaffung eines islamischen Staates auf. Ihm war aus persönlicher Erfahrung bewusst, dass jene Orientierung auf die Machtfrage und das Motivieren der islamischen Umma, ihre Verbindung mit dem Islam über eine Veränderung der politischen Ordnung zu erreichen, zwangsläufig in den Konflikt führt und der Umma keine innere Besinnung bringt. Nuri stellte dem die Strategie der "muslimischen Gesellschaft» entgegen. Diese verstand er als einen Verhaltensrahmen der Menschen in ihrer persönlichen Sphäre sowie zu anderen Elementen der Gesellschaft in Übereinstimmung mit den 
Regeln des Islams. Das zu gewährleisten, so Nuri, erfordere weder eine Veränderung der verfassungsmäßigen Ordnung, noch die Aufnahme illegaler politischer Betätigung, weil ein starker säkularer Rechtsstaat durch die Gewährleistung der religiösen Menschenrechte seinen Bürgern die Hinwendung zur Religion ermöglicht. Von dieser Grundposition aus unterstützte Nuri den säkularen Staatscharakter, woran die PIWT auch nach Nuris Tod (2006) bis heute festhält.

Diese Abwägung zwischen Politisierung und Radikalisierung erweist sich für den Umgang mit dem politischen Islam als zentral. Unter den aktuell schwierigen sozialen Bedingungen einerseits und einer irreversiblen Islamisierung Zentralasiens andererseits ist die Radikalisierung der religiösen Sphäre das zentrale Problem, weil diese zu gewaltsamen gesellschaftlichen Konflikten, ja Religionskriegen führen kann.

\section{Auf den politischen Islam einstellen}

Nach Einschätzung der russischen Zentralasien-Expertin Irina Swjagelskaja wird sich der Einfluss des politischen Islam in den Staaten Zentralasiens ausweiten und es werden neue Formen seiner Interaktion mit der säkularen Macht auftreten. Weil der Islam der wichtigste Faktor des nationalen und politischen Lebens bleibt, ist mit seiner Schwächung nicht zu rechnen. Diese neue Qualität des islamischen Faktors wird die Parameter des politischen Raums Zentralasien mitbestimmen und ist sowohl von den regionalen Herrschaftsträgern als auch in der europäischen Politik in Rechnung zu stellen. Die wichtigste Auswirkung für die säkularen Herrschaftsträger ist, dass die Ausübung politischer Macht in Zentralasien künftig untrennbar mit dem Faktor Islam verbunden sein und somit die Anpassung an diesen und dessen Vertreter zur Bedingung ihres politischen Überlebens wird. In dieser neuen Lage erschöpfen sich auch die gewohnten Kontrollinstrumente und die Herrschaftsressource Repression erweist sich als ineffektiv.

Auch in Europa (und den USA) wird die zentrale Bedeutung eines demokratischen Umgangs mit dem Faktor Islam in Zentralasien noch zu wenig erkannt. Auch sollte die Erfahrung nicht ausgeblendet werden, die westliche Staaten im arabischen Raum machen: Ohne die Kooperation mit islamischen Organisationen, Bewegungen und Parteien gelingt keine Stabilisierung und wird auch die Wahrung westlicher Interessen zukünftig nicht möglich sein. Obgleich Zentralasien allmählich wieder in den islamischen Gesamtraum hineinwächst, wäre es falsch, den zentralasiatischen politischen Islam mit dem gleichzusetzen, was wir gemeinhin unter "politischem Islam" verstehen. Noch ist ein Zeitfenster offen, um mit seinen Anhängern einen kooperativen Weg zu einem ausstrahlungsfähigen Modell neuer Beziehungen zu finden. Denn noch erfreuen sich in Zentralasien die säkulare Lebensweise und die säkularen Regierungen der Sympathie beträchtlicher Bevölkerungsteile. Auch fühlt sich die islamische Elite mit ihren nationalen Staaten verbunden und ist an guten Beziehungen zu Europa interessiert. Die islamische Politisierung befindet sich noch in einem frühen Stadium, was die Möglichkeiten ihres Missbrauchs zu extremistischen Zielen vorläufig noch in Grenzen hält. Auch unterscheidet sich die Mehrzahl der Verfechter des politischen Islam noch von denjenigen in anderen Regionen der islamischen Welt. In der sowjetischen Periode genossen sie eine weltliche Bildung, wurden mit europäischer Philosophie und Kultur, Rationalismus und Dialektik vertraut gemacht. Diese günstigen Voraussetzungen für ein konstruktives Verhältnis werden in einer Generation nicht mehr gegeben sein. Es sollte Europa gelingen, diese besondere Situation zu nutzen.

\section{Über den Autor:}

Dr. Arne C. Seifert ist Zentralasienberater des Zentrums für OSZE-Forschung (CORE) des Instituts für Friedensforschung und Sicherheitspolitik an der Universität Hamburg (IFSH).

\section{Lesetipps:}

- Arne C. Seifert/Esen Usubaliev, Relations between the State and the Muslim Community in Central Asia: Overview, Analysis, Practical Co-operation in Kyrgyzstan, in: OSCE Yearbook 2010, Baden-Baden, $164 \mid 72=$ http:// www.core-hamburg.de/documents/yearbook/english/10/SeifertUsubaliev-en.pdf

- CORE/PSIO, Confidence-Building Measures adopted by the participants of an informal secular-Islamic dialogue in Tajikistan; Dokument (Englisch/Russisch/Deutsch); CORE Working Paper 12 (2003) = http://www.core-hamburg. de/documents/CORE_Working_Paper_12.pdf

- Edren Tatari, Renat Shaykhutdinov, State Response to Religious Revivalism in Post-Soviet Central Asia, in: European Journal of Economic and Political Studies 3(2010)2, S. 85-110. Herunter zu laden von: http://ejeps.fatih.edu. tr/?journal,10 


\section{Zentralasien im Human Development Index}

Grafik 1: Human Development Index - Werte für 2010 und 2011

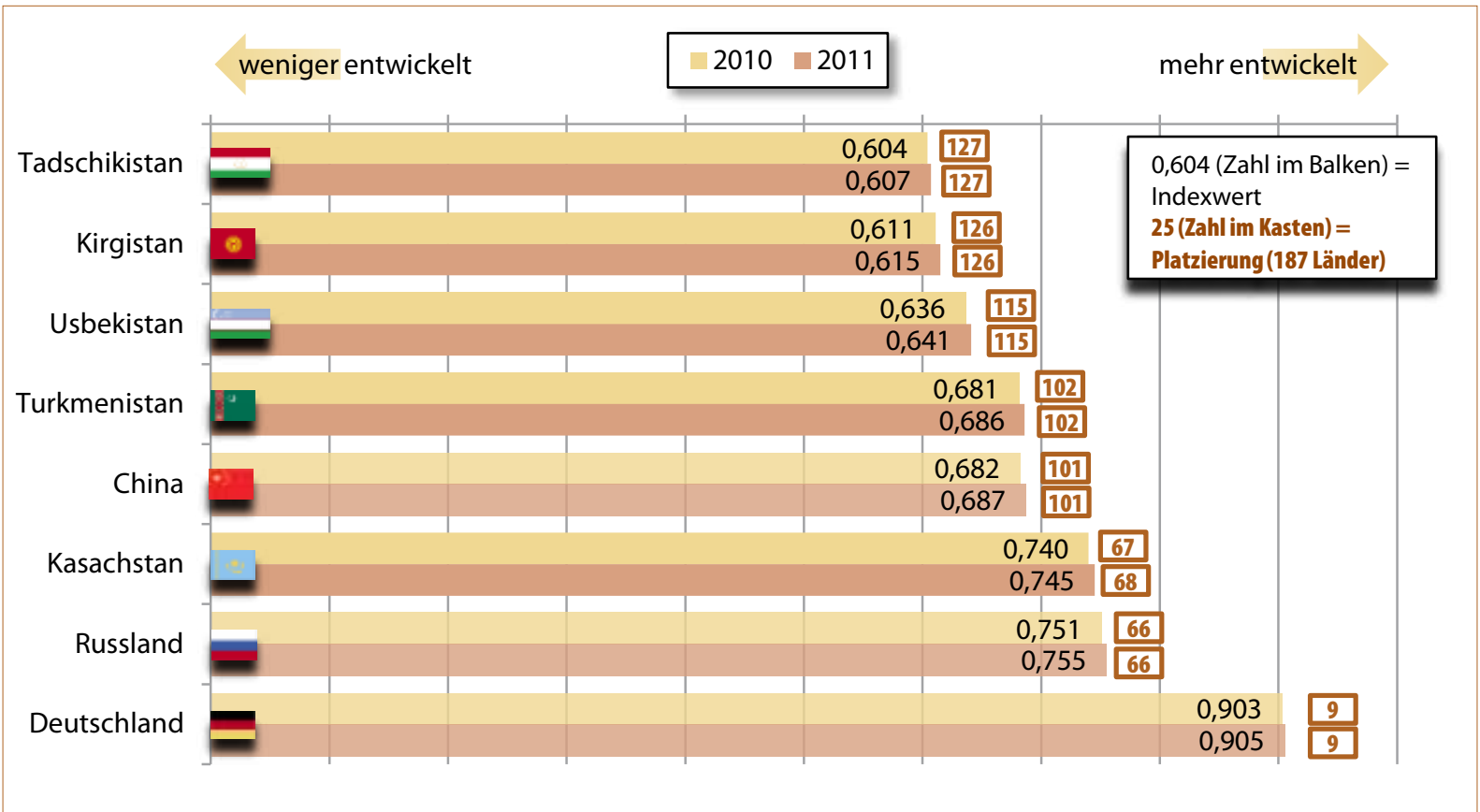

Quelle: http://hdr.undp.org/en/media/HDR_2011_EN_Table2.pdf

Grafik 2: HDI Ranking unter Berücksichtigung von Ungleichheit

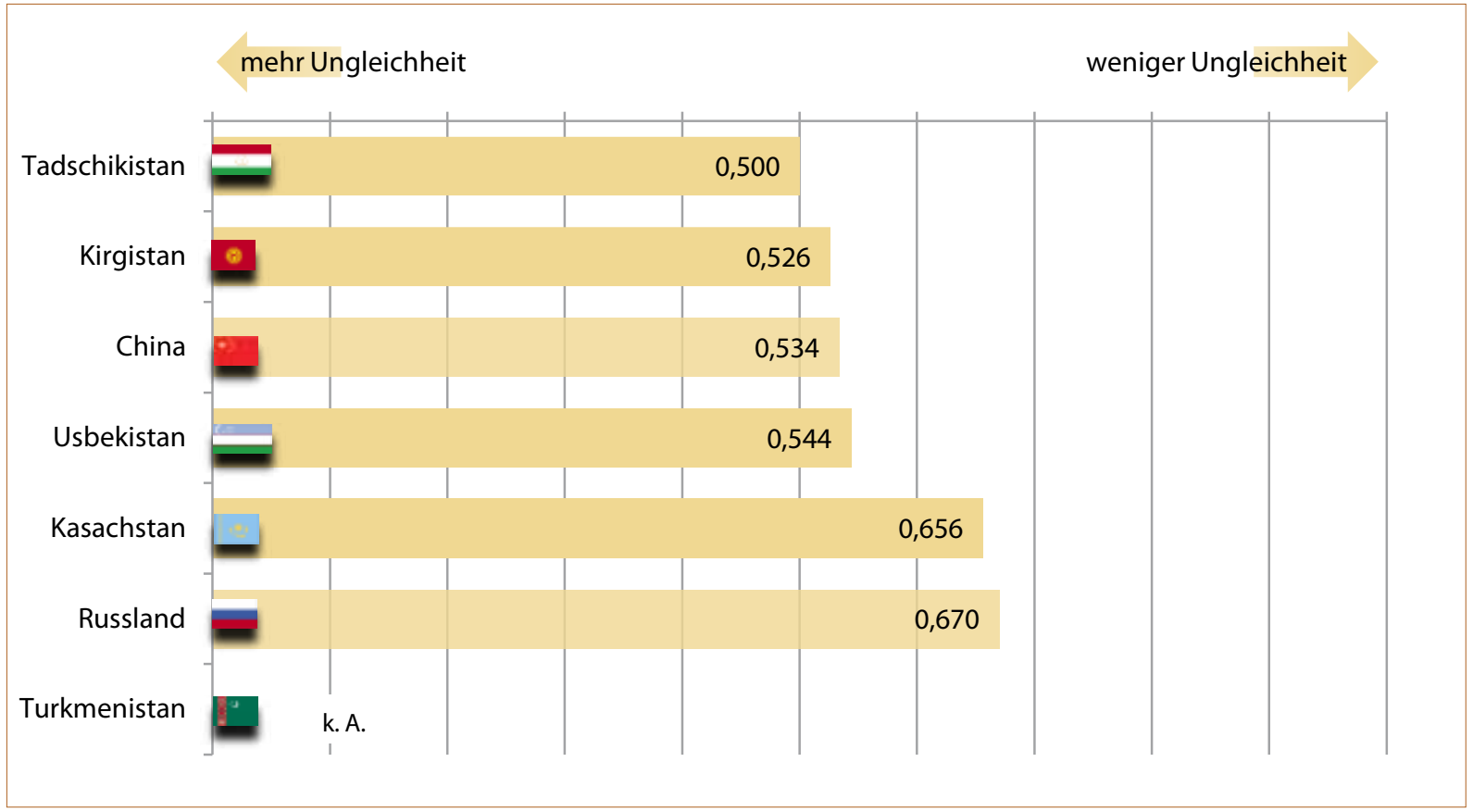

Angaben zwischen Wert "1 " für größtmögliche Gleichheit und " 0 « für größtmögliche Ungleichheit

Quelle: http://hdr.undp.org/en/media/HDR_2011_Statistical_Tables.xls 


\section{Ausgewählte sozioökonomische Grunddaten}

Grafik 3: BIP pro Kopf in den Ländern Zentralasiens für 2011 in US-Dollar. Angaben der Weltbank

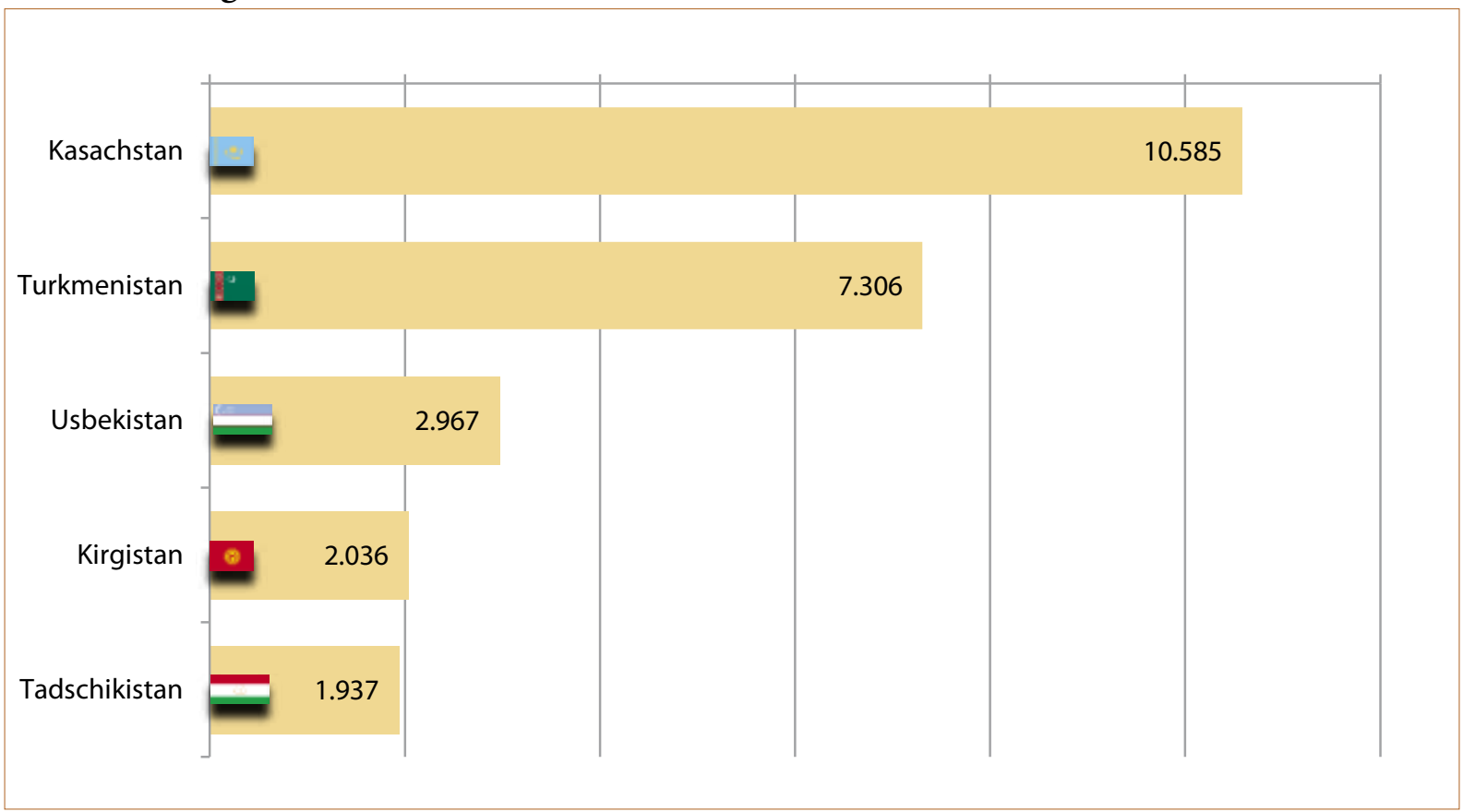

Quelle: http://hdr.undp.org/en/media/HDR_2011_Statistical_Tables.xls

Grafik 4: Entwicklung des BIP pro Kopf für die Länder Zentralasiens in US-Dollar. Kennzahlen der EBRD für die Jahre 2000, 2005, 2009

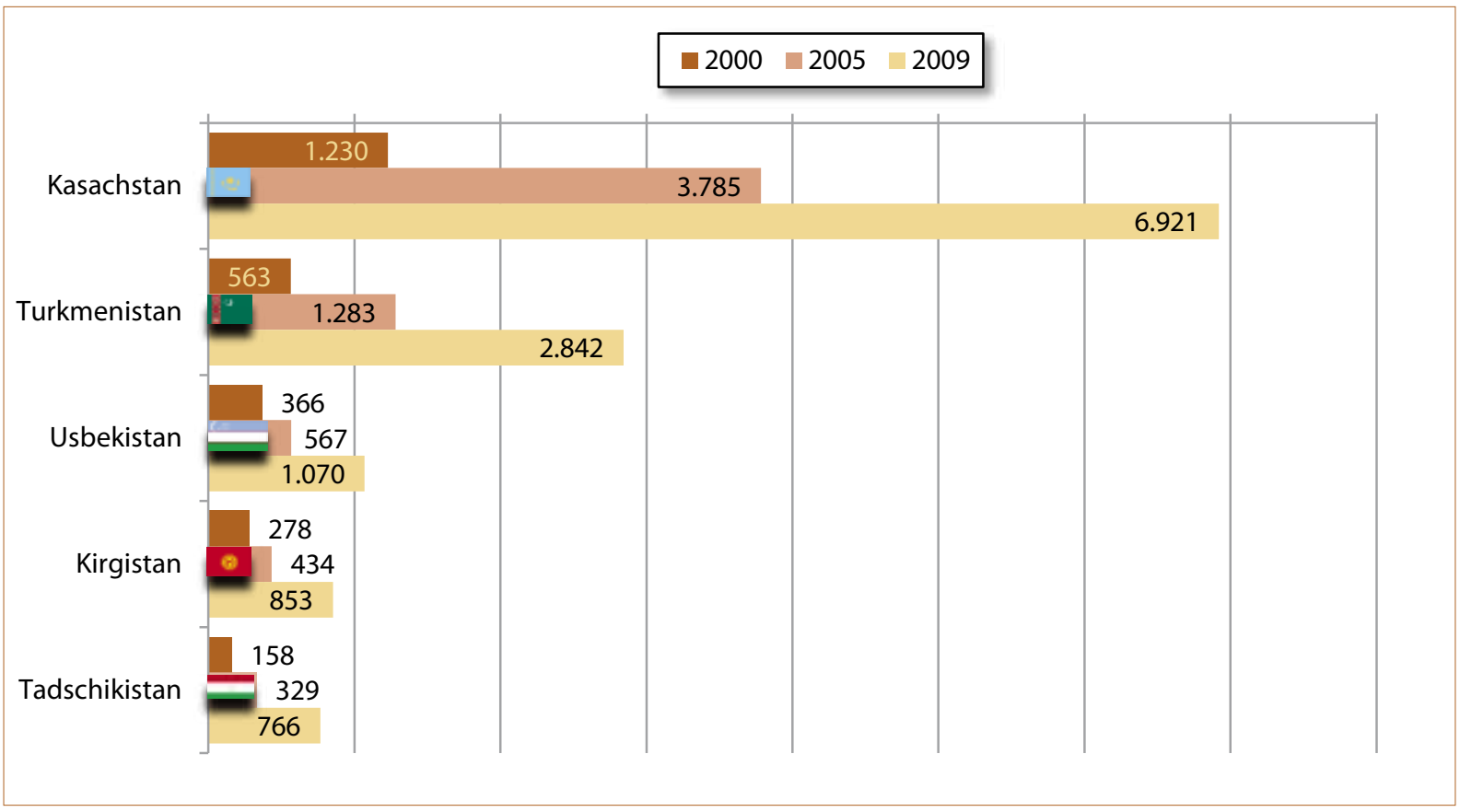


Grafik 5: Urbanisierungsgrad

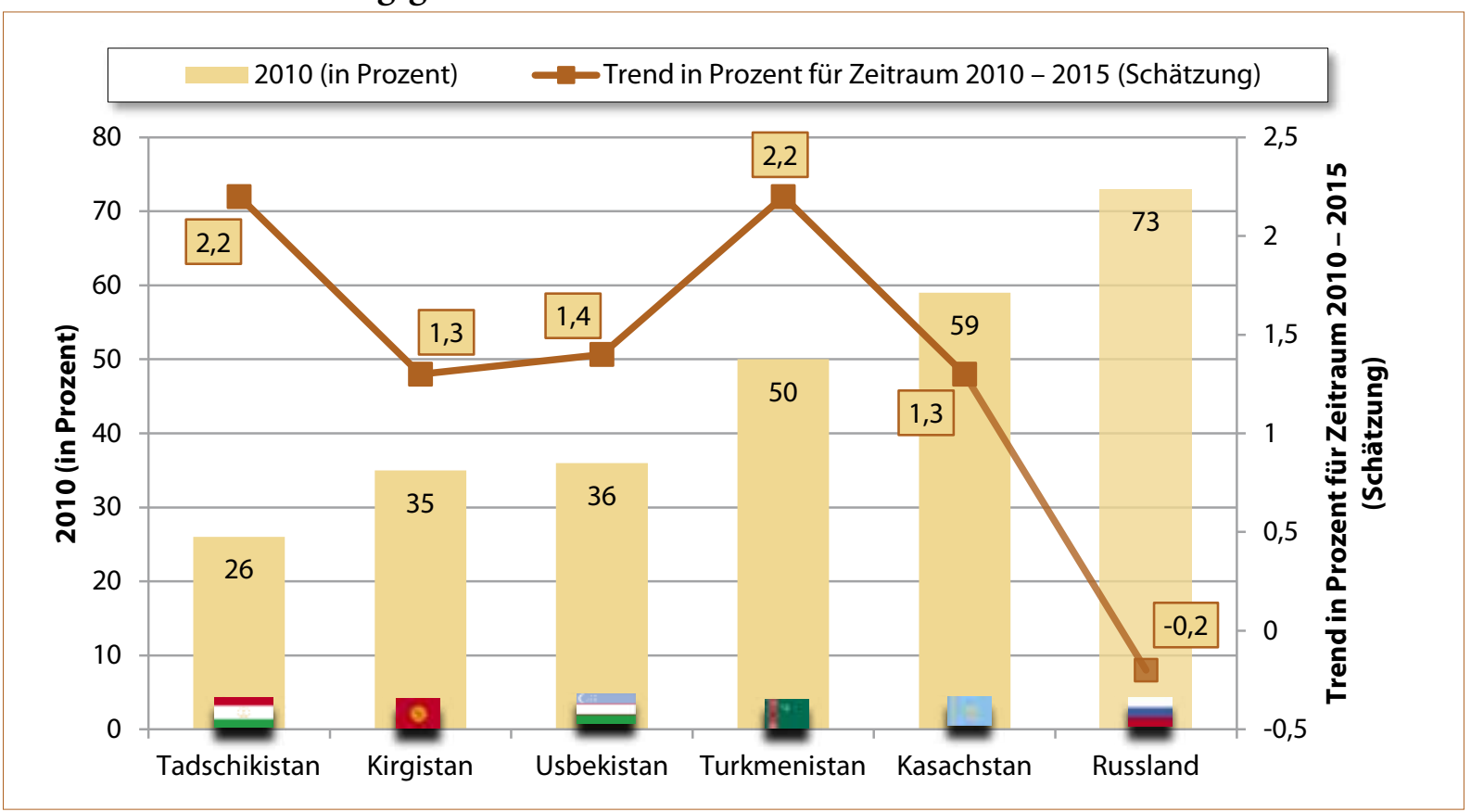

Quelle: CIA - The World Factbook; https://www.cia.gov/library/publications/the-world-factbook/wfbExt/region_cas.html

Grafik 6: Durchschnittsalter und Anteil der Bevölkerung unter 15 Jahren

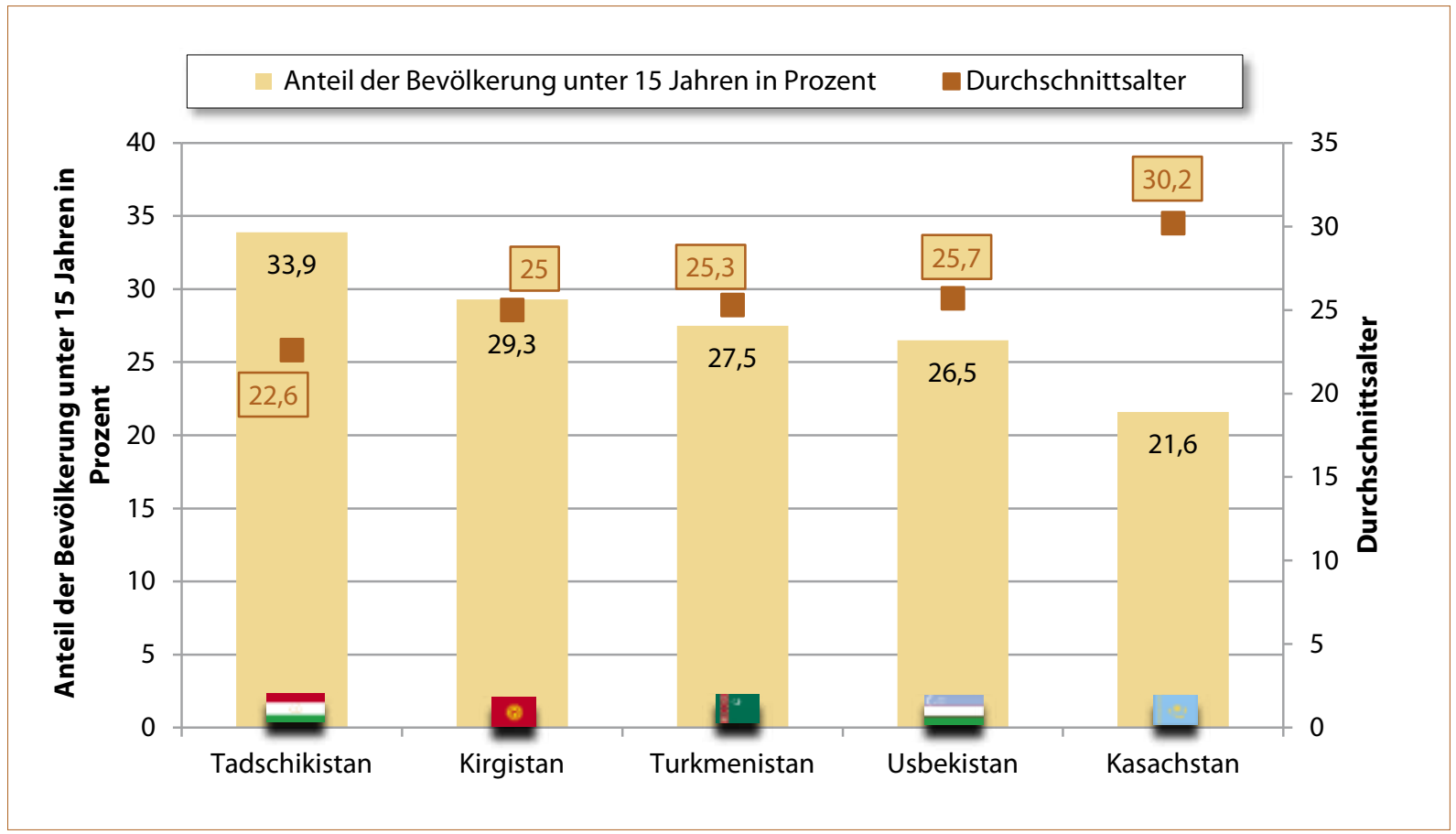

Quelle: CIA - The World Factbook; https://www.cia.gov/library/publications/the-world-factbook/wfbExt/region_cas.html 
Grafik 7: Geburtenrate pro 1.000 Einwohner und Bevölkerungswachstumsrate in Prozent, 2011

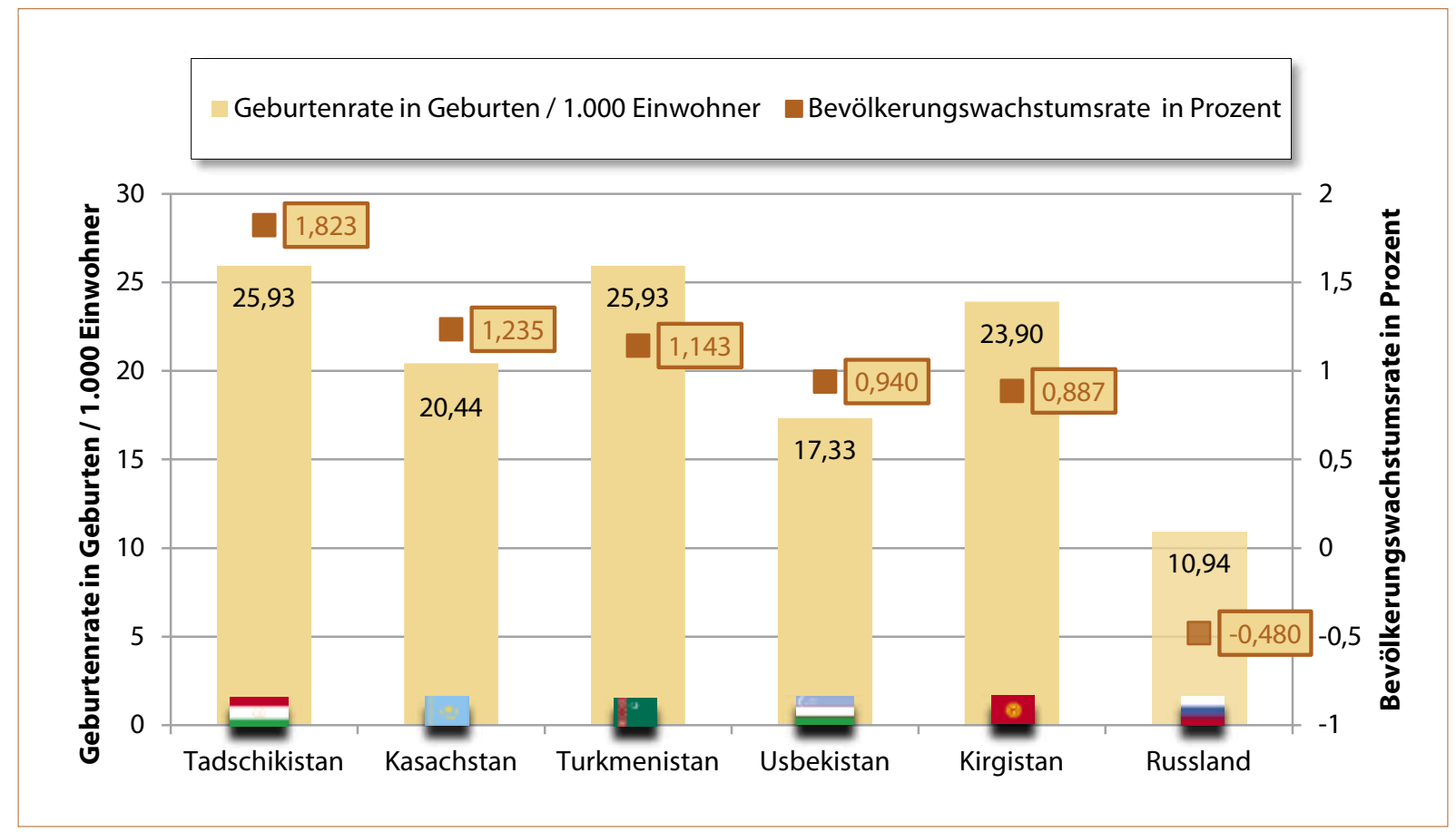

Quelle: CIA - The World Factbook; https://www.cia.gov/library/publications/the-world-factbook/wfbExt/region_cas.html

\section{Grafik 8: Anzahl der Studierenden}

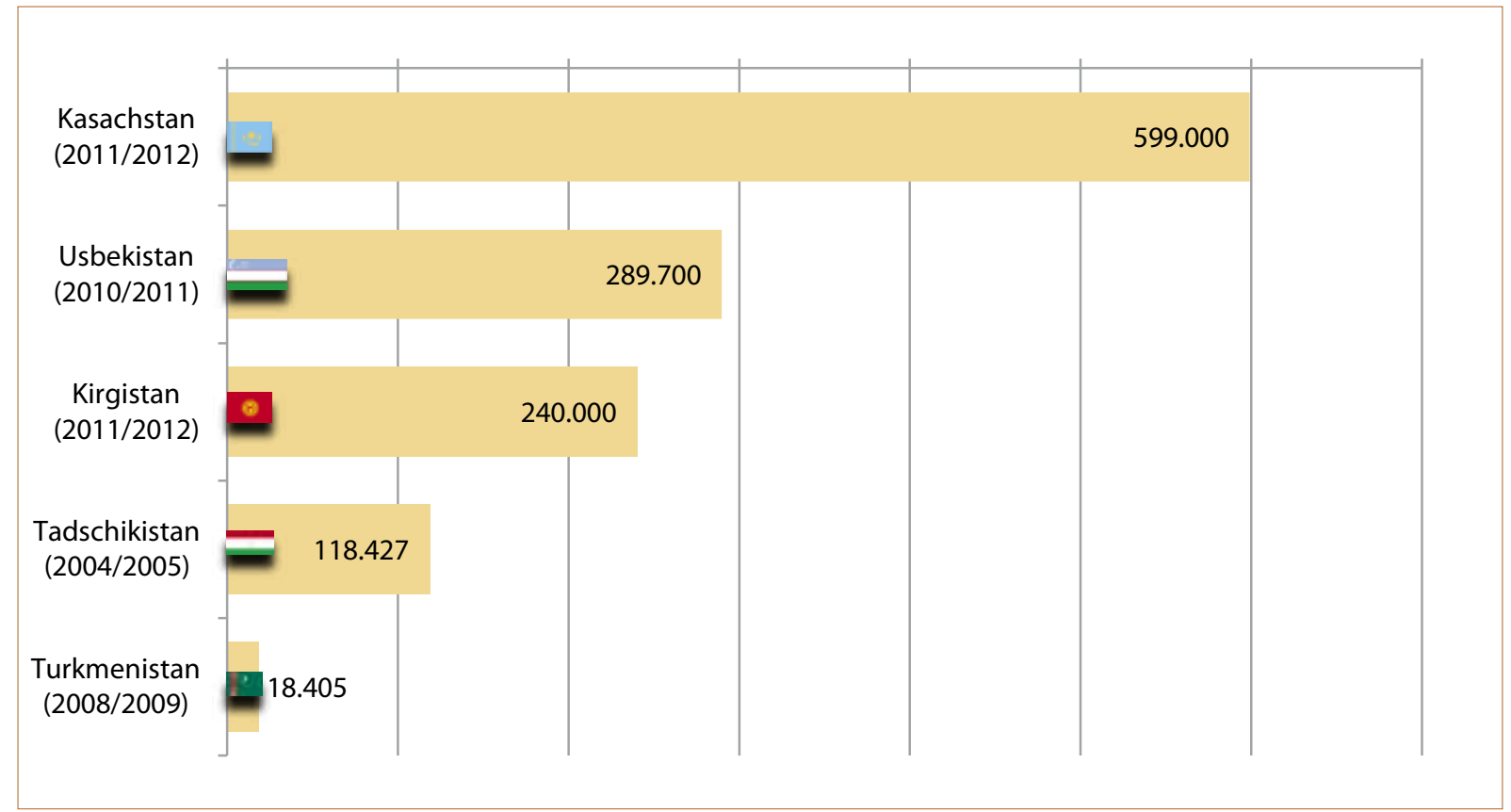

Quellen: http://univision.kz/blog/309-v-kazahstane-izbytok-studentov.html, http://www.vb.kg/news/society/2012/03/09/181565_v_kyrgyzsta ne_14_stydentov_obychautsia_na_budjetnoy_osnove.html, http://www.tajik-gateway.org/index.phtml?lang=ru\&id=4334, http://eacea.ec.euro pa.eu/tempus/participating_countries/reviews/turkmenistan_review_of_higher_education.pdf, http://www.universityfairs.com/fairs/educa tion-and-career-fair-uzbekistan-2013-4305 


\section{Religiöse Orientierung in Tadschikistan}

\section{Tabelle 1: Ergebnisse einer Meinungsumfrage zur religiösen Orientierung der Bevölkerung Tadschikistans}

\begin{tabular}{|c|c|c|}
\hline & \multicolumn{2}{|c|}{ Zustimmung (in \%) } \\
\hline Die Evolutionstheorie Darwins erklärt die Entstehung des Lebens auf der Erde & 8 & \\
\hline Die Welt und der Mensch wurden in antiker Zeit erschaffen & 55 & \\
\hline Ich bekenne mich zum Islam & 98 & \\
\hline Ich bekenne mich zum sunnitischen Islam der hanafitischen Schule & 80 & \\
\hline Ich bekenne mich zur Schia & 6 & \\
\hline Anhänger der Schia sind Muslime & 72 & \\
\hline Anhänger der Schia sind keine Muslime & 12,8 & \\
\hline $\begin{array}{l}\text { Ich gehe mindestens einmal pro Woche in die Moschee } \\
\text { (Altersgruppe bis } 22 \text { Jahre) }\end{array}$ & 33 & \\
\hline Ich gehe mehrmals pro Woche in die Moschee (Altersgruppe bis 22 Jahre) & 16 & \\
\hline Ich möchte in einem islamischen Staat leben & 7 & \\
\hline Ich möchte in einem säkularen Staat leben & 65 & \\
\hline Ich bin für die Anwendung der Scharia in häuslichen Angelegenheiten & $25-30$ & \\
\hline
\end{tabular}

Anmerkung: Das Institut Schark befragte im Frühjahr 20121500 Respondenten aus allen Gebieten Tadschikistans

Quelle: http://rus.ozodi.org/content/poll_religious_activity_tajikistan_/24559752.html 


\section{Vom 19. Mai bis zum 22. Juni 2012}

\section{Kasachstan}

\begin{tabular}{|c|c|}
\hline 19.5 .2012 & $\begin{array}{l}\text { Russische und kasachische Menschenrechtsaktivisten gründen in Almaty das Internationale Komitee zum } \\
\text { Schutz politischer Häftlinge und verfolgter Aktivisten Kasachstans. }\end{array}$ \\
\hline 21.5 .2012 & $\begin{array}{l}\text { Präsident Nursultan Nasarbajew kommt im Rahmen des 5. Astana Wirtschaftsforums mit dem Präsidenten } \\
\text { von Total E\&P, Yves-Louis Darricarrere, und dem Präsidenten von General Electric Transportation, Lorenzo } \\
\text { Simonelli, zu Gesprächen über die weitere Zusammenarbeit zusammen. }\end{array}$ \\
\hline 21.5 .2012 & $\begin{array}{l}\text { Ein Gericht in Aktau (Gebiet Mangistau) verurteilt vier im Zusammenhang mit den Ereignissen an der Zug- } \\
\text { station Schetpe (Gebiet Mangistau) am 17.12.2011 Angeklagte wegen Unruhestiftung zu mehrjährigen Haft- } \\
\text { strafen, mehrere weitere werden amnestiert bzw. rehabilitiert. }\end{array}$ \\
\hline 22.5 .2012 & $\begin{array}{l}\text { Der stellvertretende Verteidigungsminister und Stabschef der Streitkräfte Kasachstans, Saken Schassusakow, } \\
\text { wird neuer Stabschef im Hauptquartier der CSTO. }\end{array}$ \\
\hline 22.5 .2012 & $\begin{array}{l}\text { Im Rahmen der 25. Sitzung des Rats ausländischer Investoren in Astana kommt Präsident Nasarbajew mit } \\
\text { dem Präsidenten der Europäischen Bank für Wiederaufbau und Entwicklung, Thomas Mirow, zu Gesprä- } \\
\text { chen über die weitere bilaterale Zusammenarbeit zusammen. }\end{array}$ \\
\hline 22.5 .2012 & $\begin{array}{l}\text { Das Notstandsministerium berichtet, dass im nördlichen Gebiet Kostanai } 543 \text { der vom Aussterben bedroh- } \\
\text { ten Saiga-Antilopen aus unbekannter Ursache verendet sind. }\end{array}$ \\
\hline 23.5 .2012 & $\begin{array}{l}\text { In seiner Rede auf dem 5. Astana Wirtschaftsforum kündigt Präsident Nasarbajew an, dass } 86 \text { Mrd. US- } \\
\text { Dollar aus den Gas- und Öleinnahmen bei der Nationalbank als Schutz vor einer weiteren globalen Finanz- } \\
\text { krise eingesetzt werden sollen. }\end{array}$ \\
\hline 23.5 .2012 & $\begin{array}{l}\text { Präsident Nasarbajew kommt im Rahmen des 5. Astana Wirtschaftsforums mit dem ehemaligen deutschen } \\
\text { Bundespräsidenten Horst Köhler, Vertretern internationaler Organisationen und einer Gruppe Nobelpreis- } \\
\text { trägern sowie mit dem Vorstandsvorsitzenden von Exxon Mobile, Rex Tillerson, zusammen. }\end{array}$ \\
\hline 23.5 .2012 & $\begin{array}{l}\text { Der stellvertretende Minister für Öl und Gas, Bolat Aktschulakow, verkündet beim 5. Astana Wirtschaftsforum } \\
\text { den Plan, die Gasproduktion Kasachstans von heute } 40 \mathrm{Mrd} . \mathrm{m}^{3} \text { auf } 110 \mathrm{Mrd} . \mathrm{m}^{3} \mathrm{im} \text { Jahr } 2030 \text { zu steigern. }\end{array}$ \\
\hline 23.5 .2012 & $\begin{array}{l}\text { Präsident Nasarbajew und der türkischen Premierminister Recep Tayip Erdogan unterschreiben am Rande } \\
\text { des } 5 \text {. Astana Wirtschaftsforums mehrere Abkommen über eine engere wirtschaftliche und politische Zusam- } \\
\text { menarbeit beider Länder. }\end{array}$ \\
\hline 25.5 .2012 & $\begin{array}{l}\text { Das Nationale AIDS Präventionszentrum berichtet, dass seit Beginn der Registrierung von AIDS in Kasachstan } \\
\text { mehr als } 3.600 \text { Menschen an der Krankheit verstarben und gegenwärtig mehr als } 18.000 \text { AIDS Kranke regis- } \\
\text { triert sind. }\end{array}$ \\
\hline 26.5.2012 & $\begin{array}{l}\text { Der Pressedienst des Gebietsgerichts von Mangistau informiert, dass drei hochrangige Manager von KazMu- } \\
\text { naiGaz, darunter auch der ehemalige Sicherheitschef, Serik Gajmuchanbetow, vom Stadtgericht Schanaosen } \\
\text { wegen Unterschlagung im großen Stil zu mehrährigen Haftstrafen verurteilt wurden. }\end{array}$ \\
\hline 29.5 .2012 & $\begin{array}{l}\text { Fünf Polizeibeamte werden vom Gebietsgericht Mangistau wegen Machtmissbrauchs im Zusammenhang mit } \\
\text { den Ausschreitungen in Schanaosen zu Haftstrafen zwischen fünf und sieben Jahren verurteilt. }\end{array}$ \\
\hline 29.5 .2012 & $\begin{array}{l}\text { TengriNews berichtet, dass Kasachstan den Start russischer Raketen auf dem Weltraumbahnhof Baikonur } \\
\text { auf Grund eines Konflikts über den Aufprall von Raketentrümmern auf kasachstanischem Territorium blo- } \\
\text { ckiert. Der Streit verzögert auch den Transport europäischer Satelliten ins All. }\end{array}$ \\
\hline 29.5 .2012 & $\begin{array}{l}\text { Der russische Premier Dmitrij Medwedjew trifft bei seinem ersten Besuch als russischer Regierungschef in } \\
\text { Astana mit Präsident Nasarbajew und seinem Amtskollegen Karim Massimow zu Gesprächen über die wei- } \\
\text { tere bilaterale Zusammenarbeit zusammen. }\end{array}$ \\
\hline 29.5 .2012 & $\begin{array}{l}\text { Im Vorfeld des 4. Kongresses der Weltreligionen, der am 30.5. beginnt, trifft sich Präsident Nasarbajew mit } \\
\text { dem russischen Patriarchen Kirill. }\end{array}$ \\
\hline 30.5 .2012 & $\begin{array}{l}\text { Präsident Nasarbajew führt mit dem Generalsekretär der Weltliga der Muslime, Abdullah bin Abdul Moh- } \\
\text { sin Al-Turk, Gespräche über die weitere Kooperation. }\end{array}$ \\
\hline 30.5 .2012 & $\begin{array}{l}\text { Das Landwirtschaftsministerium berichtet, dass die jährliche Getreideausfuhr mit 10,6 Mio. t einen neuen } \\
\text { Höchststand erreicht hat. }\end{array}$ \\
\hline 31.5 .2012 & $\begin{array}{l}\text { Präsident Nasarbajew verurteilt auf dem } 4 \text {. Kongress der Weltreligionen den allgemeinen Sittenverfall und } \\
\text { brandmarkt neue Informationstechnologien als potentiell gesellschaftszersetzend. }\end{array}$ \\
\hline
\end{tabular}




\begin{tabular}{|c|c|}
\hline 31.5 .2012 & $\begin{array}{l}\text { Am durch ein Feuer zerstörten Grenzposten Arkankergen (Gebiet Almaty) werden die Leichen von } 14 \text { Grenz- } \\
\text { schützern gefunden; ein Soldat wird vermisst. }\end{array}$ \\
\hline 1.6 .2012 & $\begin{array}{l}\text { Nach Angaben des Ministers für Transport und Kommunikation, Askar Schumagalijew, haben Kasachstan } \\
\text { und Turkmenistan ein Abkommen über die Einrichtung eines neuen Eisenbahngrenzübergangs in Bola- } \\
\text { schak-Serchetjaka unterzeichnet. }\end{array}$ \\
\hline 1.6 .2012 & $\begin{array}{l}\text { KazTAG.kz berichtet, dass Kasachstan einer Erhöhung seiner Gaslieferungen an Kirgistan auf mehr als } 70 \\
\text { Mio. } \mathrm{m}^{3} \text { zum Preis von } 244 \text { US-Dollar pro } 1.000 \mathrm{~m}^{3} \text { zugestimmt hat. }\end{array}$ \\
\hline 1.6 .2012 & $\begin{array}{l}\text { Zwei Wochen nach seiner Premiere wird Sacha Baron Cohens neuer Film »The Dictator« in den Kinos Kasach- } \\
\text { stans ohne weitere Erklärungen von offizieller Seite aus dem Programm genommen. }\end{array}$ \\
\hline 4.6 .2012 & $\begin{array}{l}\text { Ein Gericht in Schanaosen verurteilt } 13 \text { der } 37 \text { wegen der Ereignisse in Schanaosen angeklagte Arbeiter zu } \\
\text { Haftstrafen von drei bis sieben Jahren wegen Anstiftung zu öffentlicher Unordnung. Während der Richter } \\
\text { die Urteile verkündet, kommt es im Saal zu Tumulten. }\end{array}$ \\
\hline 4.6.2012 & Angesichts des tragischen Vorfalls am Grenzposten Arkenkergen besteht am 4. Juni Staatstrauer. \\
\hline 5.6 .2012 & $\begin{array}{l}\text { BBC berichtet, dass die NATO ein Abkommen zum Transit ihrer militärischen Güter aus Afghanistan mit } \\
\text { Kasachstan, Kirgistan und Usbekistan geschlossen hat. }\end{array}$ \\
\hline 5.6 .2012 & $\begin{array}{l}\text { Die Kulturminister Irans und Kasachstans, Seyyed Mohammad Hosseini und Darchan Mynbai, verabreden } \\
\text { bei einem Treffen in Peking eine engere Zusammenarbeit. }\end{array}$ \\
\hline 5.6.2012 & $\begin{array}{l}\text { Der noch vermisste Grenzschutzsoldat des Arkankergen Grenzpostens, an dem in der Woche zuvor } 14 \text { seiner } \\
\text { Kollegen tot aufgefunden waren, meldet sich bei der Polizei. }\end{array}$ \\
\hline 6.6 .2012 & $\begin{array}{l}\text { Der Menschenrechtsaktivist und Gewerkschaftler Tachir Muchamedschanow wird in der Stadt Schachtinsk } \\
\text { (Gebiet Karaganda) tot aufgefunden. Er hatte für die Rechte der Arbeiter in den Kohleminen gestritten. }\end{array}$ \\
\hline 6.6.2012 & $\begin{array}{l}\text { Präsident Nasarbajew trifft zur Teilnahme am SCO-Gipfel in Peking ein und wird dort vom Vorsitzenden } \\
\text { der VR China, Hu Jintao, empfangen. Beide verabreden eine Intensivierung der bilateralen Zusammenarbeit. }\end{array}$ \\
\hline 7.6.2012 & $\begin{array}{l}\text { Der Nachrichtensprecher des bekannten Fernsehsenders Kanal 31, Wladislaw Dlinnow, weigert sich, eine } \\
\text { Meldung über das nach seiner Meinung gefälschte Geständnis des überlebenden Grenzsoldaten des Postens } \\
\text { Arkankergen über die Ermordung seiner } 14 \text { Kollegen zu verlesen und kündigt seine Stelle. }\end{array}$ \\
\hline 7.6.2012 & $\begin{array}{l}\text { Präsident Nasarbajew und der russische Präsident Wladimir Putin unterzeichnen in Astana mehrere Abkom- } \\
\text { men, darunter eine Ergänzung des Vertrags über Freundschaft, Kooperation und wechselseitige Hilfe sowie } \\
\text { ein Abkommen zur gemeinschaftlichen Entwicklung kleiner Kraftwerke. }\end{array}$ \\
\hline 12.6.2012 & $\begin{array}{l}\text { Die Zeitung Wremja berichtet, dass Verteidigungsminister Adilbek Schaksybekow in Jerewan und Rom } \\
\text { Abkommen über die militärische Zusammenarbeit Kasachstans mit Armenien und Italien unterzeichnet hat. }\end{array}$ \\
\hline 13.6.2012 & Astana präsentiert in Paris seine Bewerbung für die Ausrichtung der Expo-2017. \\
\hline 13.6.2012 & $\begin{array}{l}\text { Der Direktor des Grenzschutzdiensts und stellvertretende Chef des nationalen Sicherheitskomitees, Nurschan } \\
\text { Myrsalijew, tritt wegen des Vorfalls am Grenzposten Arkankergen zurück. Übergangsweise übernimmt Tur- } \\
\text { ganbek Stambekow seine Aufgaben. }\end{array}$ \\
\hline 13.6.2012 & $\begin{array}{l}\text { Der Zolldienst des Gebiets Mangistau berichtet, dass an der Grenze zu Usbekistan auf Grund neuer usbeki- } \\
\text { scher Gütertransitbestimmungen über } 170 \text { Güterwaggons aufgehalten werden. }\end{array}$ \\
\hline 13.6.2012 & $\begin{array}{l}\text { Der das Massaker am Grenzposten Arkankergen überlebende Grenzschützer Wladislaw Tschelach weist die } \\
\text { gegen ihn erhobenen Mordvorwürfe zurück. }\end{array}$ \\
\hline 13.6.2012 & $\begin{array}{l}\text { Die Nachrichtenagentur Kazinform meldet, dass die staatliche Agentur für religiöse Angelegenheiten im ers- } \\
\text { ten Quartal } 201234 \text { Internetseiten mit extremistischem Inhalt identifiziert und an die Generalstaatsanwalt- } \\
\text { schaft weitergeleitet hat. }\end{array}$ \\
\hline 14.6.2012 & $\begin{array}{l}\text { Die russischen Nachrichtenagentur Regnum berichtet, dass Usbekistan Kasachstan den Transport militäri- } \\
\text { scher Ausrüstung für ein Manöver der SCO in Tadschikistan über sein Territorium verweigert. }\end{array}$ \\
\hline 14.6.2012 & $\begin{array}{l}\text { Außenminister Jerschan Kasychanow kommt in Kabul mit dem afghanischen Präsidenten Hamid Karsai zu } \\
\text { Gesprächen über die weitere bilaterale Zusammenarbeit zusammen. Kasychanow teilt mit, dass Kasachstan } \\
\text { den afghanischen Sicherheitskräften ab } 2015 \text { finanzielle Unterstützung zukommen lassen wird. }\end{array}$ \\
\hline 14.6.2012 & $\begin{array}{l}\text { Die Nachrichtenagentur Interfax Kasachstan meldet, dass eine unabhängige Kommission zur Untersuchung } \\
\text { der Vorfälle am Grenzposten Arkankergen eingerichtet werden soll. }\end{array}$ \\
\hline
\end{tabular}




\begin{tabular}{|l|l|}
\hline 15.6.2012 & $\begin{array}{l}\text { Der bekannte Regisseur und Theaterdirektor Bolat Atabajew wird in Almaty von Mitarbeitern des Nationalen } \\
\text { Sicherheitskomitees verhaftet. Ihm wird vorgeworfen, soziale Unruhe im Zusammenhang mit den Schanao- } \\
\text { sen-Geschehnissen geschürt zu haben. Gleichzeitig wird der Jugendaktivist Schanbolat Mamai festgenommen. }\end{array}$ \\
\hline 15.6.2012 & 24 ausländische, illegal operierende Glücksspielwebsites werden geschlossen. \\
\hline 18.6.2012 & Russland kann ab sofort wieder Raketenstarts vom Weltraumbahnhof Baikonur vornehmen. \\
\hline 19.6.2012 & $\begin{array}{l}\text { 11 Grenzschutzsoldaten des Postens Tersairyk (Gebiet Ost-Kasachstan) verlassen ihre Station und werden } \\
\text { erst am nächsten Tag in einem benachbarten Dorf gefunden. Sie geben an, vor den Schikanen ihrer Vorge- } \\
\text { setzten geflohen zu sein. }\end{array}$ \\
\hline 20.6 .2012 & $\begin{array}{l}\text { Der Menschenrechtsbeauftragte der Bundesregierung, Markus Löning, und der Regisseur Volker Schlön- } \\
\text { dorff rufen die Führung Kasachstans auf, Bolat Atabajew umgehend freizulassen. }\end{array}$ \\
\hline 21.6.2012 & $\begin{array}{l}\text { Die UN verurteilen Kasachstans Entscheidung über die Auslieferung von 29 usbekischen Asylsuchenden } \\
\text { nach Usbekistan. Kasachstan rechtfertigt die Entscheidung mit dem Hinweis auf eine angebliche Mitglied- } \\
\text { schaft der Männer in einer terroristischen Vereinigung. }\end{array}$ \\
\hline $\begin{array}{l}\text { Drei Angehörige des Grenzschutzkommandos am Posten Tersairyk werden wegen des Verdachts der Miss- } \\
\text { handlung ihrer Kollegen verhaftet. }\end{array}$ \\
\hline 22.6 .2012 & $\begin{array}{l}\text { Während eines Treffens mit Vertretern kleiner und mittlerer Unternehmen setzt Präsident Nasarbajew das } \\
\text { Ziel, ihren Anteil am BIP bis 2020 40\% am BIP zu steigern. }\end{array}$ \\
\hline 22.6.2012 & \begin{tabular}{l} 
Amnesty International ruft die kasachstanische Führung auf, Bolat Atabajew umgehend freizulassen. \\
\hline
\end{tabular}
\end{tabular}

\section{Kirgistan}

\begin{tabular}{|c|c|}
\hline 20.5.2012 & $\begin{array}{l}\text { Bei einer Massenschlägerei zwischen kirgisischen und usbekischen Arbeitsmigranten in Moskau werden zwei } \\
\text { Beteiligte so schwer verletzt, dass sie in ein Krankenhaus eingeliefert werden müssen. }\end{array}$ \\
\hline 21.5 .2012 & $\begin{array}{l}\text { Das Bronzedenkmal zur Erinnerung an den } 2009 \text { in Almaty ermordeten kirgistanischen Journalisten Gen- } \\
\text { nadij Pawljuk wird in der Nacht in Bischkek gestohlen. }\end{array}$ \\
\hline 21.5 .2012 & $\begin{array}{l}\text { Nach Angaben der russischen Zentralbank haben kirgisische Arbeitsmigranten } 2011 \text { 1,4 Mrd. US-Dollar aus } \\
\text { Russland in ihre Heimat überwiesen. }\end{array}$ \\
\hline 22.5.2012 & $\begin{array}{l}\text { Außenminister Ruslan Kasakbajew hält sich zur Teilnahme an der Afghanistan-Sondersitzung des Nato- } \\
\text { Gipfels in Chicago auf. }\end{array}$ \\
\hline 23.5 .2012 & $\begin{array}{l}\text { Die Nachrichtenagentur 24.kg meldet, dass die ehemalige Präsidentin Rosa Otunbajewa in London am jähr- } \\
\text { lichen EBRD-Gipfel teilnimmt und bei dieser Gelegenheit die Öffentlichkeit in Großbritannien über die } \\
\text { aktuellen Entwicklungen in Kirgistan informiert. }\end{array}$ \\
\hline 23.5 .2012 & $\begin{array}{l}\text { Die kirgisische Regierung und die Nato unterzeichnen während des Nato-Gipfels in Chicago ein Abkom- } \\
\text { men über den Transit von Frachtgut der ISAF über das Territorium Kirgistans. }\end{array}$ \\
\hline 23.5.2012 & $\begin{array}{l}\text { Nach Angaben der staatlichen Agentur für Architektur und Bau sind mehr als } 70 \% \text { der Wasserleitungen des } \\
\text { Landes völlig desolat, für ihre Rekonstruktion sind zwischen } 10 \text { und } 23 \text { Mrd. Som ( } 212-487 \text { Mio. US-Dollar) } \\
\text { zu veranschlagen; die Regierung hat bereits Abkommen zur Finanzierung mit ADB und Weltbank geschlossen. }\end{array}$ \\
\hline 23.5 .2012 & $\begin{array}{l}\text { Innenminister Sarylbek Rysalijew berichtet vor dem Parlament, dass für die Ergreifung von Ex-Präsident } \\
\text { Kurmanbek Bakijew, seinen Sohn, seine Brüder und einige weitere Personen eine Belohnung von } 100.000 \\
\text { US-Dollar ausgesetzt wurde. }\end{array}$ \\
\hline 23.5 .2012 & $\begin{array}{l}\text { Nach Angaben von CADAP (Central Asia Drug Action Program) sind in Kirgistan offiziell } 10.000 \text { Drogen- } \\
\text { abhängige registriert. }\end{array}$ \\
\hline 23.5 .2012 & $\begin{array}{l}\text { Premierminister Omurbek Babanow unterzeichnet eine Vereinbarung zur Intensivierung der technischen und } \\
\text { ökonomischen Zusammenarbeit mit China. }\end{array}$ \\
\hline 25.5 .2012 & $\begin{array}{l}\text { Fergananews.com meldet unter Berufung auf die Nachrichtenagentur KirTag, dass insgesamt } 67 \text { der im } \\
\text { Zusammenhang mit Organisation und Teilnahme an den ethnischen Zusammenstößen in Osch } 2010 \text { Ver- } \\
\text { urteilten über Folter bei der Ermittlung berichten. }\end{array}$ \\
\hline 28.5 .2012 & $\begin{array}{l}\text { Das Außenministerium der RF teilt sein Befremden über Äußerungen des US-Botschafters in Moskau mit, } \\
\text { der am 25.5. vor Studenten gesagt hatte, dass Russland Kirgistan bestochen habe, damit es auf eine Schlie- } \\
\text { ßung von Manas dränge. Das US-Außenministerium erklärt, die russische Seite habe Botschafter Michael } \\
\text { McFaul missverstanden. }\end{array}$ \\
\hline
\end{tabular}




\begin{tabular}{|c|c|}
\hline 29.5 .2012 & $\begin{array}{l}\text { Mehr als } 200 \text { Personen demonstrieren vor dem Parlament für eine Abschaffung der gegenwärtigen Verfas- } \\
\text { sung. An ihre Stelle solle ein Grundgesetz treten, das einen vom Volk gewählten Präsidenten vorsieht, aber } \\
\text { weder ein Parlament, noch eine Regierung oder Parteien. }\end{array}$ \\
\hline 29.5 .2012 & $\begin{array}{l}\text { Nach Angaben von Vize-Premier Dschoormat Otorbajew hat die türkische Fluggesellschaft Turkish Airlines } \\
\text { ihre Pläne, einen Flug Istanbul-Bischkek-Ulan-Bator einzurichten ad acta gelegt, nachdem Mitarbeiter des } \\
\text { kirgisischen Ministeriums für Transport und Kommunikation auf ihrer Forderung von Freiflügen beharrten. }\end{array}$ \\
\hline 30.5 .2012 & $\begin{array}{l}\text { Präsident Almasbek Atambajew unterzeichnet eine Ergänzung des Gesetzes über Öl und Gas, die es der Regie- } \\
\text { rung erlaubt den Export von Öl, Gas und ihren Produkten für sechs Monate zu verbieten. }\end{array}$ \\
\hline 30.5 .2012 & $\begin{array}{l}\text { Nach Angaben des Russischen Föderalen Dienstes für Statistik erreichte der Handel zwischen Kirgistan und } \\
\text { Russland in der ersten Hälfte } 2012 \text { einen Umfang von } 352 \text { Mio. US-Dollar und ist damit im Vergleich zum } \\
\text { Vorjahreszeitraum um 21,4\% bzw. } 62 \text { Mio. US-Dollar gestiegen. }\end{array}$ \\
\hline 30.5 .2012 & $\begin{array}{l}\text { Ca. } 500 \text { Bewohner der in der Umgebung der Kumtor-Goldmine liegenden Dörfer blockieren die Zugangs- } \\
\text { straße und fordern, dass } 70 \% \text { der Einnahmen aus der Mine in Kirgistan bleiben sollen und eine Umwelt- } \\
\text { prüfung wiederholt werden solle. }\end{array}$ \\
\hline 30.5 .2012 & $\begin{array}{l}\text { Der Eigentümer der kirgisischsprachigen Oppositionszeitung Maidan.kg, Nurgasy Anarkulow, wird verhaf- } \\
\text { tet. Die Gründe sind nicht bekannt. }\end{array}$ \\
\hline 31.5 .2012 & $\begin{array}{l}\text { Premierminister Babanow begibt sich zu einem Arbeitsbesuch nach Südkorea, wo er u. a. mit seinem Amts- } \\
\text { kollegen Kim Hwang-Sik zusammenkommen wird. }\end{array}$ \\
\hline 4.6.2012 & $\begin{array}{l}\text { Präsident Atambajew unterzeichnet das neue Gesetz über friedliche Versammlungen, in dem verschiedene } \\
\text { Versammlungsformen voneinander abgegrenzt und Verhalten und Rechte von Organisatoren, Teilnehmern, } \\
\text { Behörden und Sicherheitskräften definiert werden. }\end{array}$ \\
\hline 4.6.2012 & $\begin{array}{l}\text { Premierminister Babanow hält sich zu einem offiziellen Besuch in der Türkei auf, wo er mit seinem Amtskol- } \\
\text { legen Recep Tayip Erdogan, Präsident Abdullah Gül u. a. zusammentreffen wird. }\end{array}$ \\
\hline 4.6.2012 & $\begin{array}{l}\text { AKIpress meldet, dass Ex-Präsidentin Rosa Otunbajewa auf dem Global Summit of Women in Athen mit } \\
\text { dem International Award of Leadership ausgezeichnet wurde. }\end{array}$ \\
\hline 4.6.2012 & $\begin{array}{l}\text { Die Zolldienste Kirgistans und Kasachstans vereinbaren eine vereinfachte Abfertigung kasachstanischer und } \\
\text { kirgisischer Staatsbürger an der gemeinsamen Grenze. }\end{array}$ \\
\hline 5.6.2012 & $\begin{array}{l}\text { Präsident Atambajew und sein chinesischer Amtskollege Hu Jintao unterzeichnen in Peking eine Deklaration } \\
\text { über die Vertiefung der bilateralen Beziehungen und ein Abkommen über technisch-ökonomische Zusam- } \\
\text { menarbeit. Atambajew hält sich zur Teilnahme am SCO-Gipfel in China auf. }\end{array}$ \\
\hline 5.6 .2012 & $\begin{array}{l}\text { Der 10.6. wird mit Dekret des Präsidenten zum nationalen Gedenktag für die Opfer der tragischen Ereig- } \\
\text { nisse von Osch und Dschalalabad im Juni } 2010 .\end{array}$ \\
\hline 5.6 .2012 & $\begin{array}{l}\text { Nach Angaben des Sozialfonds erhalten } 92 \% \text { der Rentner Altersbezüge, die unter der offiziellen Armuts- } \\
\text { grenze von } 4.166 \text { Som ( } 88,2 \text { US-Dollar) liegen. }\end{array}$ \\
\hline 5.6 .2012 & $\begin{array}{l}\text { Die Sicherheitskräfte in der Stadt Osch stehen ab sofort bis Mitte Juni unter erhöhter Alarmbereitschaft, es } \\
\text { sind Spezialtruppen in der Stadt stationiert, eine Ausweispflicht wird eingeführt. }\end{array}$ \\
\hline 5.6 .2012 & $\begin{array}{l}\text { Menschenrechtler melden, dass Sicherheitskräfte versucht haben, das Haus der Familie des } 2010 \text { zu einer } \\
\text { lebenslangen Freiheitsstrafe verurteilten Menschenrechtlers Asimschan Askarow zu konfiszieren. }\end{array}$ \\
\hline 7.6.2012 & Auf dem SCO-Gipfel in Peking wird beschlossen, dass der Summit 2013 in Bischkek stattfinden soll. \\
\hline 7.6.2012 & $\begin{array}{l}\text { Eine Arbeitsgruppe aus Mitarbeitern des Komitees für nationale Sicherheit, der Generalstaatsanwaltschaft und } \\
\text { des Innenministeriums übergibt Präsident Atambajew die Ergebnisse einer Untersuchung der Ereignisse von } \\
\text { Osch 2010, die Atambajew im Februar } 2012 \text { in Auftrag gegeben hatte. Sie entspricht im Wesentlichen den } \\
\text { Ergebnissen der nationalen Untersuchungskommission, neu ist, dass sie Provokationen von Bakijew-Anhän- } \\
\text { gern, Separatisten und Kriminelle für die Ereignisse verantwortlich macht. }\end{array}$ \\
\hline 8.6.2012 & $\begin{array}{l}\text { Nach Angaben des Nationalen Statistischen Komitees hat sich das ökonomische Wachstum in den ersten } \\
\text { fünf Monaten des Jahres } 2012 \text { verlangsamt, das BIP macht mit 90.607,7 Mio. Som (1.918,7 Mio. US-Dol- } \\
\text { lar) nur 93,6\% des BIP des Vorjahreszeitraums aus. }\end{array}$ \\
\hline 8.6.2012 & $\begin{array}{l}\text { Im Vorfeld des Jahrestages von Osch berichten mehrere Medien von der schwierigen Situation der Usbeken } \\
\text { im Süden. 24.kg zitiert den Ombudsmann für Menschenrechte, Tursunbek Akun, dass Gerichte und Sicher- } \\
\text { heitskräfte sich Usbeken gegenüber gesetzlos verhalten würden. }\end{array}$ \\
\hline
\end{tabular}




\begin{tabular}{|c|c|}
\hline 8.6 .2012 & $\begin{array}{l}\text { Amnesty International kritisiert in seinem neuesten Bericht die kirgisische Führung wegen der mangelhaf- } \\
\text { ten Aufarbeitung der Vorgänge in Osch. }\end{array}$ \\
\hline 10.6.2012 & $\begin{array}{l}\text { In Osch beginnen unter Teilnahme von Präsident Atambajew, Bürgermeister Melis Myrsakmatow und wei- } \\
\text { terer hochrangiger Persönlichkeiten mehrere Gedenkveranstaltungen für die Opfer der Juni-Ereignisse } 2010 . \\
\text { Außerdem werden der Denkmalskomplex Manas und die neue, größte Moschee Kirgistans, die bis zu } 20.000 \\
\text { Gläubige fasst, offiziell eröffnet. }\end{array}$ \\
\hline 11.6.2012 & $\begin{array}{l}\text { Mehrere im Exil lebende Vertreter der Usbeken Kirgistans, unter ihnen Kadyrschan Batyrow, fordern in einer } \\
\text { aus Anlass des Jahrestages verfassten gemeinsamen Erklärung die UN und die OSZE zur genauen Beobach- } \\
\text { tung der Situation der nationalen Minderheiten und ggfs. zu Sanktionen gegen Kirgistan auf. }\end{array}$ \\
\hline 11.6.2012 & $\begin{array}{l}\text { Nach Angaben der Vorsitzenden der Assoziation der Mikrofinanzinstitute, Nargisa Scholdoschewa, waren } \\
\text { Anfang } 201271 \% \text { der Empfänger von Mikrokrediten weiblich; } 44 \% \text { der Mikrokredite gehen in die Land- } \\
\text { wirtschaft, } 27 \% \text { in Handel und Kommerz. }\end{array}$ \\
\hline 12.6.2012 & $\begin{array}{l}\text { Der indische Staatsminister für äußere Angelegenheiten, Edappakat Ahamed, erörtert in Bischkek mit Pre- } \\
\text { mierminister Babanow Fragen des bilateralen Verhältnisses und kündigt den Bau von medizinischen Ein- } \\
\text { richtungen durch indische Firmen an. }\end{array}$ \\
\hline 12.6.2012 & $\begin{array}{l}\text { In einem Bischkeker Gefängnis begeht der } 2010 \text { zu lebenslanger Haft verurteilte Gafurschan Abdurachma- } \\
\text { now Selbstmord. Der frühere Führer der usbekischen Gemeinde des Bezirks Susak (Gebiet Dschalalabad) war } \\
\text { wegen seiner Beteiligung an gewalttätigen Demonstrationen bei der Fabrik Sanpa im Juni 2010, bei denen } \\
16 \text { Personen zu Tode kamen, verurteilt worden. }\end{array}$ \\
\hline 12.6 .2012 & $\begin{array}{l}\text { Das Nationale Sicherheitskomitee ermittelt im Gebiet Naryn wegen des Verdachts der Verschickung von Schul- } \\
\text { kindern zur religiösen Ausbildung nach Bangladesh. Hinter der Aktion soll die Tablig-i Dschamaat stehen. }\end{array}$ \\
\hline 12.6.2012 & $\begin{array}{l}\text { Das Management der privaten Kabelfernsehgesellschaft Ala teilt mit, dass es in beiderseitigem Einverneh- } \\
\text { men die Übertragung des oppositionellen kasachstanischen Senders K-plus einstellt. Die Übertragung war } \\
\text { auf Drängen des offiziellen Kasachstan bereits mehrfach unterbrochen worden. }\end{array}$ \\
\hline 13.6.2012 & $\begin{array}{l}\text { Präsident Atambajew unterzeichnet Veränderungen des Gesetzes über den Wehrdienst, mit denen die } 2008 \\
\text { geschaffene Möglichkeit des Freikaufs vom Dienst bei der Armee (Preis } 12.000 \text { Som, } 254 \text { US-Dollar) abge- } \\
\text { schafft wird. }\end{array}$ \\
\hline 14.6.2012 & $\begin{array}{l}\text { In Bischkek findet die dritte Sitzung der Vorsitzenden der turksprachigen Parlamente statt. Kirgistan hat zur- } \\
\text { zeit den Vorsitz der Parlamentarischen Versammlung der turksprachigen Nationen inne. }\end{array}$ \\
\hline 14.6.2012 & $\begin{array}{l}\text { Das Parlament verabschiedet in dritter Lesung das Gesetz über Visa-Freiheit bei einem Aufenthalt bis } 60 \text { Tage } \\
\text { für Bürger von } 44 \text { Staaten, darunter auch Deutschland, Österreich und die Schweiz. }\end{array}$ \\
\hline 18.6.2012 & $\begin{array}{l}\text { Kirgisische Medien berichten, dass jährlich mehr als } 100 \text { verstorbene Kirgisen aus Russland in ihre Heimat } \\
\text { überführt werden müssen. }\end{array}$ \\
\hline 18.6.2012 & $\begin{array}{l}300 \text { Wehrdienstleistende aus Kirgistan, Kasachstan, Tadschikistan, Afghanistan und den USA nehmen an } \\
\text { dem Manöver Regionale Zusammenarbeit } 2012 \text { in Kirgistan teil. }\end{array}$ \\
\hline 19.6 .2012 & $\begin{array}{l}\text { In Bischkek demonstrieren ca. } 50 \text { Studenten vor dem Ministerium für Bildung und Wissenschaft gegen die } \\
\text { Schließung der Abteilung Journalismus an der Staatlichen Technischen Rassakow-Universität. }\end{array}$ \\
\hline 19.6.2012 & $\begin{array}{l}\text { Mehrere hundert LKW-Fahrer blockieren aus Protest gegen einen Regierungsbeschluss vom 10.5.2012, mit } \\
\text { dem die Nutzung von LKW mit einer Nutzlast von mehr als } 30 \text { t beschränkt wurde, schon den zweiten Tag } \\
\text { die Überlandstraße Osch-Bischkek. }\end{array}$ \\
\hline 19.6.2012 & $\begin{array}{l}\text { Der Sohn des Ata-Meken Parlamentsabgeordneten Abdimuchtar Mamatow, Akylbek, wird unter dem Ver- } \\
\text { dacht des Schmuggels festgenommen. Der Chef mehrerer Erdölhandelsfirmen soll einen Verlust von } 22 \text { Mio. } \\
\text { Som ( } 465.900 \text { US-Dollar) verursacht haben und geständig sein. }\end{array}$ \\
\hline 20.6.2012 & $\begin{array}{l}\text { Das Parlament verabschiedet eine Resolution, in der die Rückgabe der Gas- und Ölförderanlagen Severnyj } \\
\text { Soch und Tschongara-Galtscha (Gebiet Batken) gefordert wird. Kirgistan hatte sie } 1992 \text { Usbekistan überlas- } \\
\text { sen, obwohl sie sich auf kirgisischem Territorium befinden. }\end{array}$ \\
\hline 20.6 .2012 & $\begin{array}{l}\text { Nach Angaben von Kirgisgaz hat Kirgistan die höchsten Gaspreise in der GUS, weil es mehr als } 90 \% \text { sei- } \\
\text { nes Bedarfs importieren muss. }\end{array}$ \\
\hline 21.6.2012 & $\begin{array}{l}\text { Abgeordnete der Parlamentsfraktionen von Ar-Namys und Ata-Meken beginnen mit der Unterschriften- } \\
\text { sammlung für ein Misstrauensvotum gegen die Regierung. }\end{array}$ \\
\hline
\end{tabular}




\begin{tabular}{|l|l|}
\hline 21.6.2012 & $\begin{array}{l}\text { Die Verkündung des Urteils gegen den wegen Anheizens zwischenethnischer Feindschaft angeklagten Jour- } \\
\text { nalisten Wladimir Farafonow muss ein zweites Mal verschoben werden, da der Angeklagte nicht erscheint. } \\
\text { Sein Aufenthaltsort ist unbekannt. }\end{array}$ \\
\hline 21.6.2012 & $\begin{array}{l}\text { Nariman Tjulejew, Parlamentsabgeordneter der Ata-Dschurt-Partei und ehemaliger Bürgermeister von Bisch- } \\
\text { kek, wird am Abend unter Korruptionsverdacht festgenommen. }\end{array}$ \\
\hline
\end{tabular}

\section{Tadschikistan}

\begin{tabular}{|c|c|}
\hline 21.5.2012 & Der Chef des Grenzpostens Wachsch, Nisomiddin Jokubow, wird mit $20 \mathrm{~kg}$ Haschisch festgenommen. \\
\hline 21.5.2012 & räsident Emomali Rachmon schenkt dem Fußballklub seiner Heimatstadt Kuljab einen Bus. \\
\hline 21.5.2012 & $\begin{array}{l}\text { Nach Angaben der russischen Zentralbank haben tadschikische Arbeitsmigranten } 20112,75 \text { Mrd. US-Dol- } \\
\text { lar aus Russland in ihre Heimat überwiesen. }\end{array}$ \\
\hline 22.5 .2012 & $\begin{array}{l}\text { Außenminister Hamrochon Zarifi führt am Rande der Afghanistan-Sitzung des Nato-Gipfels in Chicago } \\
\text { eine Reihe von Gesprächen mit US-amerikanischen Politikern und Diplomaten. }\end{array}$ \\
\hline 22.5 .2012 & $\begin{array}{l}\text { Mehrere Journalistenvereinigungen äußern in einer gemeinsamen Erklärung ihre Besorgnis über die zuneh- } \\
\text { mende Zahl von tätlichen Übergriffen auf Journalisten und fordern eine genaue Untersuchung der Fälle. }\end{array}$ \\
\hline 23.5 .2012 & $\begin{array}{l}\text { Ein Sprecher der regierenden Volksdemokratischen Partei Tadschikistans kündigt an, dass die Partei Präsident } \\
\text { Rachmon als ihren Kandidaten für die im November } 2013 \text { anstehende Präsidentenwahl nominieren wird. }\end{array}$ \\
\hline 24.5 .2012 & Im Süden Moskaus wird ein Tadschike erschossen aufgefunden. \\
\hline 25.5.2012 & $\begin{array}{l}\text { Die untere Kammer des Parlaments billigt ein Gesetz, nach dem Bürger der USA, der EU und einiger süd- } \\
\text { ostasiatischer Staaten sich bis zu einen Monat visafrei in Tadschikistan aufhalten können. Das Gesetz muss } \\
\text { noch vom Senat abgesegnet werden. }\end{array}$ \\
\hline 27.5.2012 & $\begin{array}{l}\text { In Teheran beginnt die neunte Sitzung der gemeinsamen Wirtschaftskommission Iran-Tadschikistan. Nach } \\
\text { Angaben aus Teheran soll es u. a. um den Import von } 1 \mathrm{Mrd} . \mathrm{m}^{3} \text { Trinkwasser aus Tadschikistan gehen. }\end{array}$ \\
\hline 29.5 .2012 & $\begin{array}{l}\text { Der tadschikische Dienst von RFE/RL meldet, dass der Anwalt des verhafteten Geschäftsmannes Nisom- } \\
\text { chon Dschurajew, Berdikul Rasulow, unter Korruptionsverdacht festgenommen wurde. }\end{array}$ \\
\hline 30.5 .2012 & $\begin{array}{l}\text { Während ihres Treffens in Aschchabad vereinbaren der tadschikische Premierminister Akil Akilow und sein } \\
\text { kirgisischer Amtskollege Omurbek Babanow eine Reihe von gemeinsamen Infrastruktur- und Energievorha- } \\
\text { ben, u. a. den Ausbau der Verbindung Duschanbe-Jirgital-Karamik-Sarytasch zu einer internationalen Straße. }\end{array}$ \\
\hline 30.5 .2012 & $\begin{array}{l}\text { Ein Gericht im Gebiet Sogd verurteilt } 17 \text { Männer wegen Mitgliedschaft in der IMU zu Haftstrafen von neun } \\
\text { bis } 23 \text { Jahren und Konfiszierung ihres Eigentums. }\end{array}$ \\
\hline 6.6.2012 & $\begin{array}{l}\text { Asia-Plus meldet, dass durch eine Gesetzesänderung ab sofort Geldstrafen von bis zu } 4.000 \text { Somoni (840 US- } \\
\text { Dollar) gegen Personen, die im Ausland religiöse Studien, die nicht tadschikischen Gesetzen entsprechen, } \\
\text { betreiben und gegen Organisationen, die nicht gesetzeskonforme Verbindungen zu ausländischen religiösen } \\
\text { Vereinigungen aufnehmen, verhängt werden können. }\end{array}$ \\
\hline 8.6.2012 & $\begin{array}{l}\text { Im Polygon Tschoruch-Dairon beginnt eine Anti-Terrorübung der SCO unter Beteiligung von insgesamt } \\
20.000 \text { Soldaten aus Russland, China, Kasachstan, Kirgistan und Tadschikistan. }\end{array}$ \\
\hline 11.6 .2012 & $\begin{array}{l}\text { In einem Moskauer Untersuchungsgefängnis wird ein 32-jähriger tadschikischer Häftling tot aufgefunden, } \\
\text { alle Indizien sprechen für Selbstmord. }\end{array}$ \\
\hline 12.6.2012 & $\begin{array}{l}\text { Die beliebte Nachrichtenwebsite www.news.tj ist zwei Tage lang nur eingeschränkt zugänglich, weil einige } \\
\text { Provider offiziell zur Blockierung der Seite aufgefordert wurden, nicht aber der offizielle Betreiber der Seite, } \\
\text { meldet Asia-Plus. }\end{array}$ \\
\hline 13.6 .2012 & $\begin{array}{l}\text { Am späten Abend wird in Duschanbe ein Schwager Präsident Rachmons erschossen. Der } 57 \text {-jährige Chol- } \\
\text { mumin Safarow war Direktor von Tadschikles, einem staatlichen Unternehmen für Forstwirtschaft. Die } \\
\text { Ermittlungen wurden eingeleitet. }\end{array}$ \\
\hline 15.6 .2012 & $\begin{array}{l}\text { Präsident Rachmon empfängt den iranischen Vizepräsidenten Lotfollah Forouzandeh Dehkordi zu Gesprä- } \\
\text { chen über die bilaterale Zusammenarbeit. }\end{array}$ \\
\hline 15.6 .2012 & $\begin{array}{l}\text { Die iranische Aluminiumgesellschaft unterzeichnet ein Memorandum of Understanding mit Tadschikistan } \\
\text { über die Lieferung von } 100.000 \text { t Aluminiumpulver und } 100.000 \text { t Aluminiumhydrat innerhalb von } 20 \\
\text { Monaten im Wert von } 80 \text { Mio. US-Dollar. }\end{array}$ \\
\hline 15.6.2012 & $\begin{array}{l}\text { Das Oberhaus des Parlamentes beschließt die Umbenennung mehrerer Dörfer im Gebiet Chatlon, die noch } \\
\text { sowjetische Namen wie Kuibischew, Komsomol, Agronom hatten. Sie tragen nun tadschikische Namen. }\end{array}$ \\
\hline
\end{tabular}




\begin{tabular}{|l|l|}
\hline 15.6.2012 & $\begin{array}{l}\text { Die tadschikische Eisenbahngesellschaft reduziert wegen stark gesunkenen Passagieraufkommens die Zahl } \\
\text { ihrer Zugverbindungen nach Moskau von drei auf zwei Mal in der Woche, meldet Asia-Plus. Die Verbin- } \\
\text { dung Duschanbe-Saratow wird aus gleichem Grund eingestellt. Auch die Fluggesellschaften melden sinken- } \\
\text { den Passagierzahlen auf ihren Flügen nach Moskau. }\end{array}$ \\
\hline 18.6.2012 & Auf einer Baustelle im Gebiet Leningrad wird ein 26-jähriger tadschikischer Bauarbeiter ermordet aufgefunden. \\
\hline 20.6.2012 & $\begin{array}{l}\text { Das Parlament beschließt die Kürzung der Einmalzahlung, die Mitarbeiter der Sicherheitskräfte, der Justiz } \\
\text { und des Parlamentes mit Renteneintritt (oder im Fall von Invalidität oder Tod) erhalten. }\end{array}$ \\
\hline 20.6.2012 & $\begin{array}{l}\text { Die US-Behörden schränken die Aufenthaltsdauer für tadschikische Studenten, die am Work \& Travel Pro- } \\
\text { gramm teilnehmen wollen, von vier auf zwei Monate ein und verringern die Zahl der möglichen Teilnehmer. }\end{array}$ \\
\hline
\end{tabular}

\section{Turkmenistan}

\begin{tabular}{|c|c|}
\hline 19.5.2012 & $\begin{array}{l}\text { In Aschchabad wird im Rahmen der Feiern zum 20. Unabhängigkeitstag im Vergnügungspark Elem das } \\
\text { größte Riesenrad der Welt in Betrieb genommen. }\end{array}$ \\
\hline 21.5 .2012 & $\begin{array}{l}\text { Die Bezeichnung Großer Vaterländischer Krieg für die Kriegshandlungen zwischen der Sowjetunion und } \\
\text { Nazi-Deutschland von 1941-1945 wird in Turkmenistan verboten. }\end{array}$ \\
\hline 22.5 .2012 & $\begin{array}{l}\text { Der erste stellvertretende Außenminister Wepa Hajijew nimmt an der Sitzung des NATO-Rats zur Lage in } \\
\text { Afghanistan in Chicago teil. }\end{array}$ \\
\hline 22.5.2012 & $\begin{array}{l}\text { Präsident Gurbanguly Berdymuchammedow ordnet anlässlich der im April erfolgten Wahl der Stadt Mary } \\
\text { zur GUS Kulturhauptstadt die Organisation einer Konferenz »Antikes Merv - Weltkulturzentrum» für den } \\
\text { 6. Juni an. }\end{array}$ \\
\hline 23.5 .2012 & $\begin{array}{l}\text { Ein Konsortium der beiden südkoreanischen Unternehmen LG International und Hyundai Engineering Co. } \\
\text { Ltd. erhält den Auftrag zum Bau einer Raffinerie in Turkmenistan im Wert von } 530 \text { Mio. US-Dollar. Die } \\
\text { Fertigstellung ist für } 2015 \text { geplant. }\end{array}$ \\
\hline 23.5 .2012 & $\begin{array}{l}\text { Vertreter von Turkmenistan, Afghanistan, Pakistan und Indien unterzeichnen ein Abkommen über die Lie- } \\
\text { ferung turkmenischen Gases durch die geplante TAPI Gaspipeline, die alle vier Länder auf einer Strecke von } \\
1700 \mathrm{~km} \text { verbinden und ab } 201890 \text { Mio. } \mathrm{m}^{3} \text { Gas liefern soll. }\end{array}$ \\
\hline 24.5 .2012 & $\begin{array}{l}\text { Der jährliche Menschenrechtsbericht des US State Department stellt eine unverändert schlechte Menschen- } \\
\text { rechtslage in Turkmenistan (und Usbekistan) fest. }\end{array}$ \\
\hline 25.5 .2012 & $\begin{array}{l}\text { In ihrer Hauszeitung Rysgal (Erfolg) ruft die Assoziation der Industriellen und Unternehmer Turkmenistans } \\
\text { unter Leitung von Aleksandr Dadajew seit Anfang Mai wöchentlich zur Gründung einer neuen Partei auf. }\end{array}$ \\
\hline 25.5 .2012 & $\begin{array}{l}\text { Präsident Berdymuchammedow ernennt den Direktor der Staatlichen Agentur für die Nutzung fossiler Brenn- } \\
\text { stoffe, Jagschygeldi Kakajew, zum stellvertretenden Premierminister. }\end{array}$ \\
\hline 25.5 .2012 & $\begin{array}{l}\text { Präsident Berdymuchammedow unterschreibt die Dekrete zur Gründung des Turkmenischen Staatlichen Insti- } \\
\text { tuts für Öl und Gas und zur Gründung des Turkmenischen Staatlichen Instituts für Architektur und Bauwesen. }\end{array}$ \\
\hline 25.5 .2012 & $\begin{array}{l}\text { Präsident Berdymuchammedow entlässt den Minister für Öl- und Gasindustrie und Mineralressourcen, } \\
\text { Bajramgeldi Nedirow. Nachfolger wird der bisherige stellvertretende Direktor des staatlichen Gaskonzerns } \\
\text { Turkmengas, Kakageldi Abdyllajew. }\end{array}$ \\
\hline 28.5 .2012 & $\begin{array}{l}\text { Präsident Berdymuchammedow empfängt den Vize-Direktor der Weltbank für Europa und Asien, Philippe } \\
\text { Le Houérou, zu Gesprächen über die weitere Zusammenarbeit. }\end{array}$ \\
\hline 28.5.2012 & $\begin{array}{l}\text { Chronika Turkmenistana berichtet, dass der Bau neuer Denkmäler zur Erinnerung an die Opfer des zweiten } \\
\text { Weltkriegs und des Erdbebens vom 6. Oktober } 1948 \text { angeordnet wurde. Bisher erinnert nur ein Denkmal in } \\
\text { Gestalt der Mutter des verstorbenen Präsidenten Saparmurat Nijasow, Gurbansoltan-Edsche, an das Erdbeben. }\end{array}$ \\
\hline 29.5 .2012 & $\begin{array}{l}\text { Präsident Berdymuchammedow ernennt neue Direktoren für die Fernseh- und Radiosender Altyn Asyr: Turk- } \\
\text { menistan, Jaschlyk, Miras, und Turkmen Owasy. }\end{array}$ \\
\hline 29.5.2012 & $\begin{array}{l}\text { Präsident Berdymuchammedow trifft sich im Vorfeld des Treffens der GUS-Regierungschefs mit dem Sekre- } \\
\text { tär des Exekutivkomitees der GUS, Sergej Lebedjew und mit den Regierungschefs von Belarus, Kasachstan, } \\
\text { Kirgistan, Tadschikistan, Armenien, Ukraine und dem Vize-Premierminister von Aserbaidschan. }\end{array}$ \\
\hline 30.5 .2012 & $\begin{array}{l}\text { Am Rande des Treffens der GUS-Regierungschefs in Aschchabad trifft sich Präsident Berdymuchammedow } \\
\text { mit den Premierministern von Russland und Moldawien, Dmitrij Medwedjew und Vlad Filat. }\end{array}$ \\
\hline 31.5 .2012 & $\begin{array}{l}\text { In Aschchabad kommen die Premierminister der Mitgliedsländer der GUS zusammen. Bei dem Treffen wer- } \\
\text { den eine Reihe finanzwirtschaftlicher und industriepolitischer Abkommen unterzeichnet. }\end{array}$ \\
\hline
\end{tabular}




\begin{tabular}{|c|c|}
\hline 31.5 .2012 & $\begin{array}{l}\text { Präsident Berdymuchammedow und der kirgisische Premierminister Omurbek Babanow erörtern die Mög- } \\
\text { lichkeit der Eröffnung einer turkmenischen Botschaft in Bischkek und einer direkten Flugverbindung zwi- } \\
\text { schen beiden Hauptstädten. }\end{array}$ \\
\hline 1.6 .2012 & $\begin{array}{l}\text { Die turkmenischsprachige Website des Forums Ertir.com muss ihren Zugang in Turkmenistan wegen Hacker- } \\
\text { attacken für einige Tage einstellen. Gegenwärtig sind über } 8000 \text { Nutzer auf der Seite registriert. }\end{array}$ \\
\hline 4.6 .2012 & $\begin{array}{l}\text { Präsident Berdymuchammedow empfängt eine chinesische Delegation zu Gesprächen über die weitere mili- } \\
\text { tärische Zusammenarbeit. }\end{array}$ \\
\hline 6.6 .2012 & $\begin{array}{l}\text { Turkmengas und die chinesische CNPC vereinbaren, die Exportmenge turkmenischen Gases nach China in } \\
\text { Zukunft auf jährlich } 65 \mathrm{Mrd} . \mathrm{m}^{3} \text { zu steigern. }\end{array}$ \\
\hline 6.6 .2012 & $\begin{array}{l}\text { Präsident Berdymuchammedow kommt in Peking am Rande der 12. Sitzung des Rats der Staatsoberhäup- } \\
\text { ter der SCO mit dem Vorsitzenden der KP Chinas Hu Jintao und mit dem iranischen Präsidenten Mahmud } \\
\text { Ahmadinedschad zu Gesprächen über die weitere bilaterale Zusammenarbeit zusammen. }\end{array}$ \\
\hline 7.6.2012 & $\begin{array}{l}\text { Präsident Berdymuchammedow erörtert in Peking mit dem afghanischen Präsidenten Hamid Karsai die wei- } \\
\text { tere Zusammenarbeit zwischen den beiden Ländern. }\end{array}$ \\
\hline 7.6.2012 & $\begin{array}{l}\text { Bei einem Fußballspiel kommt es in Turkmenabad zu Massenausschreitungen zwischen Mannschaften und } \\
\text { Fans. }\end{array}$ \\
\hline 7.6.2012 & $\begin{array}{l}\text { AFP berichtet, dass im russischen Theater von Aschchabad mit Othello erstmals seit } 2001 \text { wieder ein klas- } \\
\text { sisches Stück aufgeführt wird. Der frühere Präsident Nijasow hatte das klassische Theater als dem Turkme- } \\
\text { nischen fremd abgeschafft. }\end{array}$ \\
\hline 8.6.2012 & $\begin{array}{l}\text { Erstmals zeigt der turkmenische Sportkanal mit der Fußball-Europameisterschaft ein internationales Sport- } \\
\text { ereignis live im Fernsehen. }\end{array}$ \\
\hline 9.6 .2012 & $\begin{array}{l}\text { Eine Delegation des Internationalen Komitees des Roten Kreuzes hält sich zu Gesprächen über die weitere } \\
\text { Kooperation in Turkmenistan auf. }\end{array}$ \\
\hline 13.6.2012 & $\begin{array}{l}\text { Präsident Berdymuchammedow empfängt den Vorsitzenden des türkischen Parlaments, Cemil Cicek, zu } \\
\text { Gesprächen über die weitere bilaterale Zusammenarbeit. }\end{array}$ \\
\hline 14.6.2012 & $\begin{array}{l}\text { Präsident Berdymuchammedow trifft den Vizepräsidenten des Unternehmens Chevron, Jay Pryor. Es werden } \\
\text { Möglichkeiten der Zusammenarbeit im Energiesektor besprochen und Chevrons Interesse am Bau der TAPI. }\end{array}$ \\
\hline 15.6.2012 & $\begin{array}{l}\text { Der Vorsitzende des türkischen Parlaments, Cemil Cicek, ruft auf der Parlamentarischen Versammlung der } \\
\text { turksprachigen Nationen in Kirgistan Turkmenistan (und Usbekistan) zum Beitritt auf. }\end{array}$ \\
\hline 20.6.2012 & $\begin{array}{l}\text { Chronika Turkmenistan berichtet, dass dem turkmenischen Botschafter in Aserbaidschan eine Protestnote } \\
\text { wegen der turkmenischen Erkundungsarbeiten an dem zwischen beiden Ländern umstrittenen kaspischen } \\
\text { Gasfeld Kjapas überreicht wurde. }\end{array}$ \\
\hline 20.6.2012 & $\begin{array}{l}\text { Präsident Berdymuchammedow nimmt in Brasilien an der Konferenz Rio+20 teil und trifft sich am Rande der } \\
\text { Veranstaltung mit dem bulgarischen Präsidenten Rosen Plewnelijew, dem tadschikischen Präsidenten Emomali } \\
\text { Rachmon und dem russischen Premier Medwedjew zu Gesprächen über die weitere bilaterale Zusammenarbeit. }\end{array}$ \\
\hline
\end{tabular}

\section{Usbekistan}

\begin{tabular}{|l|l|}
\hline 19.5.2012 & $\begin{array}{l}\text { Vertreter des usbekisch-koreanischen Joint Ventures Uz-Kor Gas Chemical unterzeichnen Kreditverträge in } \\
\text { Höhe von 2,54 Mrd. US-Dollar für die Implementierung eines integrierten Gasförder- und Ölverarbeitungs- } \\
\text { komplexes im Ust-Jurt Gebiet. }\end{array}$ \\
\hline 19.5.2012 & $\begin{array}{l}\text { Der usbekische Dienst von RFE/RL meldet, dass im Falle der Ermordung des ehemaligen Ministers für Kul- } \\
\text { tur und Sport, Anwar Dschabborow, mehrere Verdächtige verhaftet worden sind. }\end{array}$ \\
\hline 21.5.2012 & $\begin{array}{l}\text { Aus Menschrechtskreisen wird bekannt, dass am 19.5. in Karschi ein Überfall auf die Menschenrechtlerin } \\
\text { Gulschan Karajewa verübt wurde. }\end{array}$ \\
\hline 21.5.2012 & $\begin{array}{l}\text { In der schwedischen Presse werden genauere Details über die beiden verhafteten Attentäter auf Imam Obidchon } \\
\text { Nasarow bekannt. Es handelt sich demnach um ein Ehepaar aus Usbekistan, das im Februar 2011 nach Schwe- } \\
\text { den eingereist war und in Malmö lebte. Nasarow befindet sich seit dem Überfall im Februar 2012 im Koma. }\end{array}$ \\
\hline 21.5.2012 & $\begin{array}{l}\text { Die japanische Entwicklungshilfeorganisation JICA gewährt dem Taschkenter Alischer Nawoi Theater einen } \\
\text { Kredit von 400.000 US-Dollar zur Verbesserung der technischen Ausstattung. }\end{array}$ \\
\hline 21.5.2012 & $\begin{array}{l}\text { Nach Angaben der russischen Zentralbank haben usbekische Arbeitsmigranten 2011 4,9 Mrd. US-Dollar } \\
\text { aus Russland in ihre Heimat überwiesen. }\end{array}$ \\
\hline
\end{tabular}




\begin{tabular}{|c|c|}
\hline 22.5 .2012 & $\begin{array}{l}\text { Außenminister Abdulassis Kamilow und Verteidigungsminister Kabul Berdijew nehmen an der Sondersit- } \\
\text { zung über Afghanistan auf dem Nato-Gipfel in Chicago teil. }\end{array}$ \\
\hline 22.5 .2012 & $\begin{array}{l}\text { Uznews.net berichtet, dass durch das Eingreifen von Menschenrechtsaktivisten am letzten Wochenende Unru- } \\
\text { hen auf dem Uriksor Basar von Taschkent abgewendet werden konnten. Anlass waren Probleme beim Ver- } \\
\text { kauf einer großen Ladung von besonders billigem Zucker. }\end{array}$ \\
\hline 22.5 .2012 & In Petersburg wird ein 21-jähriger Usbeke nachts auf offener Straße erstochen. \\
\hline 23.5 .2012 & $\begin{array}{l}\text { Laut einer Meldung von Uznews hat die Menschenrechtlerin Gulschan Karajewa, auf die vier Tage zuvor ein } \\
\text { Überfall verübt wurde, Präsident Karimow in einem Brief aufgefordert, sie und ihren Sohn am 1. Juni zu } \\
\text { erschießen, um sie vor den ständigen Übergriffen der Sicherheitskräften zu schützen. So könnten auch ihre } \\
\text { sterblichen Überreste in der Heimat bleiben. }\end{array}$ \\
\hline 23.5 .2012 & $\begin{array}{l}\text { GULI, das Label von Präsidententochter Gulnora Karimowa, tritt als Sponsor einer Gala in Monaco auf, an } \\
\text { der Prinz Albert II. von Monaco und der ehemalige US Präsident und Ehemann der US-Außenministerin, } \\
\text { Bill Clinton, als Gastgeber auftreten. }\end{array}$ \\
\hline 23.5 .2012 & $\begin{array}{l}\text { In der Taschkenter Tageszeitung Toshkent Haqiqati (Taschkenter Wahrheit) erscheint ein Artikel, der die } \\
\text { Leser vor Informationsattacken aus dem Ausland warnt, die sich durch Kritik und Fehlinformation der natür- } \\
\text { lichen Ressourcen des Landes bemächtigen wollten. }\end{array}$ \\
\hline 24.5 .2012 & $\begin{array}{l}\text { Fergananews.com meldet, dass in diesem Jahr in der autonomen Republik Karakalpakstan schon drei Minis- } \\
\text { ter und vier Bürgermeister wegen Wirtschaftsvergehen ihres Amtes enthoben wurden. }\end{array}$ \\
\hline 24.5 .2012 & $\begin{array}{l}\text { Der Prozess gegen die Rentnerin Tamara Talaschmanowa, die am } 26.4 .2012 \text { festgenommen worden war, weil } \\
\text { sie starke Beruhigungsmittel mit sich führte, wird eingestellt. Beobachter führen dies auf den Druck russi- } \\
\text { scher Diplomaten und Medien zurück. Talaschmanowa ist russische Staatsbürgerin. Ihr hätte eine Freiheits- } \\
\text { strafe von fünf bis sieben Jahren gedroht. }\end{array}$ \\
\hline 24.5 .2012 & $\begin{array}{l}\text { Sacha Baron Cohens neuester Film, The Dictator, wird in Usbekistan zwar in Kinos gezeigt, allerdings in } \\
\text { stark zensierter Fassung, meldet RFE/RL. }\end{array}$ \\
\hline 25.5 .2012 & $\begin{array}{l}\text { Im neuesten Jahresbericht des US State Department zur globalen Menschenrechtssituation wird Usbekistan } \\
\text { wieder neben Turkmenistan, China, Syrien, Iran, Nordkorea und Belarus besonders negativ bewertet. }\end{array}$ \\
\hline 25.5.2012 & $\begin{array}{l}\text { Die Menschenrechtlerin Jelena Urlajewa hat sich erneut und freiwillig in psychiatrische Behandlung bege- } \\
\text { ben. Sie war erst zwei Wochen zuvor entlassen worden und hatte kurz darauf wieder mit der Menschenrechts- } \\
\text { arbeit begonnen. }\end{array}$ \\
\hline 28.5 .2012 & $\begin{array}{l}\text { Die Intercontinental-Hotelgruppe gibt ihren Rückzug aus Usbekistan bekannt. Sie hatte seit } 1997 \text { gemein- } \\
\text { sam mit der usbekischen Regierung das einzige Fünf-Sterne-Hotel Taschkents bzw. Usbekistans betrieben. }\end{array}$ \\
\hline 29.5 .2012 & $\begin{array}{l}\text { HRW kritisiert im Vorfeld der jährlichen Konferenz der International Labour Organisation (ILO) in Genf in } \\
\text { einem offenen Brief an EU-Außenkommissarin Catherine Ashton und den dänischen Außenminister Villy } \\
\text { Sovndal, dass die EU viel zu lange Kinderarbeit, Folter und Übergriffe gegen Menschenrechtsaktivisten hin- } \\
\text { genommen habe und fordert dazu auf, unmissverständlich auf die Einreiseerlaubnis für eine ILO-Mission } \\
\text { und ein Ende der Folter zu drängen. }\end{array}$ \\
\hline 30.5 .2012 & $\begin{array}{l}\text { Der Vizepräsident der Weltbank für Europa und Zentralasien, Phillipe Le Houérou, erklärt am Ende sei- } \\
\text { nes viertägigen Usbekistanbesuches, dass das Land gute ökonomische Kennziffern habe, aber noch großer } \\
\text { Reformbedarf bestehe. }\end{array}$ \\
\hline 31.5 .2012 & $\begin{array}{l}\text { Die Chefs des chinesischen und usbekischen Generalstabs, Chen Bingde und Machmudow, stimmen bei } \\
\text { einem Treffen in Taschkent darin überein, dass eine Intensivierung der bilateralen militärischen Zusammen- } \\
\text { arbeit erstrebenswert sei. }\end{array}$ \\
\hline 31.5 .2012 & $\begin{array}{l}\text { In einer von Fergananews.com publizierten Presseerklärung der indischen Textilgesellschaft Spentex Indus- } \\
\text { tries Ltd. zum Bankrott von Spentex Toshkent Toytepa wird die usbekische Regierung beschuldigt, Verein- } \\
\text { barungen nicht eingehalten zu haben und es werden } 100 \text { Mio. US-Dollar Kompensation für die entstande- } \\
\text { nen Verluste von Usbekistan gefordert. }\end{array}$ \\
\hline 31.5 .2012 & $\begin{array}{l}\text { Die russische Nachrichtenagentur Interfax meldet, dass Sicherheitskräfte in Taschkent zwei junge Männer } \\
\text { unter dem Verdacht festgenommen haben, } 14 \text { Frauen vergewaltigt, beraubt und ermordet zu haben. }\end{array}$ \\
\hline 1.6.2012 & $\begin{array}{l}\text { Eine Mission des IMF empfiehlt nach Ende ihres Usbekistanaufenthaltes vom 21.-25. Mai der Regierung, } \\
\text { Maßnahmen zur Inflationsbekämpfung zu ergreifen und zuverlässige Statistiken zu veröffentlichen. }\end{array}$ \\
\hline 1.6 .2012 & $\begin{array}{l}14 \text { Lehrer in der Stadt Parkent (Gebiet Taschkent) werden entlassen, weil sie enge Angehörige haben, die aus } \\
\text { politischen Gründen verhaftet sind oder weil sie eine Moschee besucht haben. }\end{array}$ \\
\hline
\end{tabular}




\begin{tabular}{|c|c|}
\hline 4.6 .2012 & $\begin{array}{l}\text { Der russische Präsident Wladimir Putin besucht während seiner Asienreise als erstes Taschkent und führt dort } \\
\text { Gespräche mit seinem Amtskollegen Karimow, dem er versichert, dass Usbekistan für Russland ein Schlüs- } \\
\text { selland sei. Im MoU wird Usbekistans Beitritt zur Freihandelszone der GUS Staaten angekündigt. }\end{array}$ \\
\hline 4.6.2012 & $\begin{array}{l}\text { Im Gebiet Dschissak beginnt eine gemeinsame Anti-Terror-Übung von Sicherheitskräften aus Usbekistan, } \\
\text { Kasachstan und Kirgistan. }\end{array}$ \\
\hline 5.6 .2012 & $\begin{array}{l}\text { Präsident Karimow trifft zu einem Staatsbesuch in China ein. Nach Treffen mit der chinesischen Staatsspitze } \\
\text { wird er am 6. und 7.6. am SCO-Gipfel in Peking teilnehmen. }\end{array}$ \\
\hline 5.6.2012 & $\begin{array}{l}\text { Das Innenministerium bestätigt die neuen Regeln für die Registrierung von Ausländern und staatenlosen } \\
\text { Personen in Stadt und Gebiet Taschkent. }\end{array}$ \\
\hline 6.6.2012 & $\begin{array}{l}\text { Das erste staatliche Fernsehprogramm warnt mit einer Sendung über illegale Arbeitsmigration die Bürger des } \\
\text { Landes vor der Ausreise als Gastarbeiter. }\end{array}$ \\
\hline 6.6 .2012 & $\begin{array}{l}\text { Ein Sprecher des Verteidigungsministeriums gibt vor Journalisten bekannt, dass Usbekistan nicht an der Anti- } \\
\text { Terror-Übung der SCO vom 7.-14.6.2012 in Tadschikistan teilnehmen wird. }\end{array}$ \\
\hline 8.6.2012 & $\begin{array}{l}\text { Nach Angaben von Uzreport.com wurden während des Chinabesuches Präsident Karimows Wirtschaftsab- } \\
\text { kommen im Gesamtumfang von 5,2 Mrd. US-Dollar zwischen Usbekistan und China unterzeichnet. }\end{array}$ \\
\hline 8.6 .2012 & $\begin{array}{l}\text { Fergananews.com meldet, dass Präsidententochter Gulnora Karimowa mit dem SCO-Preis für humanitäre } \\
\text { Zusammenarbeit ausgezeichnet wurde. }\end{array}$ \\
\hline 8.6 .2012 & $\begin{array}{l}\text { Einige Tage nachdem Menschenrechtler die Entlassung von } 14 \text { Lehrern in der Stadt Parkent bekannt gemacht } \\
\text { hatten, wurden alle Betroffenen wieder eingestellt. }\end{array}$ \\
\hline 11.6 .2012 & $\begin{array}{l}\text { Anders als bisher müssen ausländische LKW-Fahrer ab sofort im Voraus Einfuhrgenehmigungen nach Usbe- } \\
\text { kistan beantragen. Bislang war das an der Grenze möglich. }\end{array}$ \\
\hline 12.6 .2012 & $\begin{array}{l}\text { Der stellvertretende US Secretary of State, William Burns, erörtert während seines Taschkentbesuches mit } \\
\text { Präsident Karimow Fragen der bilateralen Zusammenarbeit. }\end{array}$ \\
\hline 12.6 .2012 & $\begin{array}{l}\text { Fergananews.com meldet, dass nun in Moskau eine usbekischsprachige Zeitung für Arbeitsmigranten mit } \\
\text { dem Namen Ozbegim (Mein Usbekistan) erscheint. }\end{array}$ \\
\hline 12.6.2012 & $\begin{array}{l}\text { In Taschkent werden mehrere Anhänger der Bewegung Birdamlik (Solidarität) bei dem Versuch, vor der Bot- } \\
\text { schaft Kirgistans zum Gedenken an die Ereignisse in Osch } 2010 \text { zu demonstrieren, festgenommen. Sei wer- } \\
\text { den am gleichen Abend wieder freigelassen, nachdem einige von ihnen zur Zahlung hoher Geldstrafen ver- } \\
\text { urteilt worden waren. }\end{array}$ \\
\hline 13.6.2012 & $\begin{array}{l}\text { In Taschkent findet die sechste Sitzung der Zwischenregierungskommission Usbekistan-Schweiz für Handel } \\
\text { und wirtschaftliche Zusammenarbeit statt. }\end{array}$ \\
\hline 13.6.2012 & $\begin{array}{l}\text { Vor einem Bezirksgericht im Gebiet Taschkent beginnt der Prozess gegen die Menschenrechtlerin Gulnasa } \\
\text { Juldaschewa. Der Studentin wird Erpressung vorgeworfen. }\end{array}$ \\
\hline 14.6.2012 & $\begin{array}{l}\text { Nach Angaben von Fergananews.com stellt das traditionsreiche Tschkalow-Flugzeugwerk in Taschkent die } \\
\text { Produktion von Flugzeugen ein und die Produktion auf andere Erzeugnisse um. }\end{array}$ \\
\hline 14.6.2012 & $\begin{array}{l}\text { Verschiedene Medien berichten von Gerüchten, dass sowohl der Vertreter der usbekischen Tochterfirma der } \\
\text { russischen Mobilfunkfirma MTS, Bekzod Achmedow, wie auch der CEO von Carlsberg Uzbekistan, Jewge- } \\
\text { nij Schetschenko, das Land verlassen hätten. Carlsberg soll sich aus Usbekistan zurückziehen. }\end{array}$ \\
\hline 15.6 .2012 & Der Bürgermeister von Buchara, Chajit Komilow, wird unter Korruptionsverdacht verhaftet. \\
\hline 15.6 .2012 & $\begin{array}{l}\text { Präsident Karimow unterzeichnet das Dekret zur Ernennung von Normomin Tschorijew zum Gouverneur des } \\
\text { Gebietes Suchandarja. Tschorijew war bereits seit Dezember } 2011 \text { kommissarischer Gouverneur des Gebietes. }\end{array}$ \\
\hline 15.6 .2012 & $\begin{array}{l}\text { UzDaily.uz meldet unter Berufung auf das staatliche Komitee für Statistik, dass Energieträger im ersten Quar- } \\
\text { tal } 2012 \text { mit 33,6\% einen im Vergleich zu } 2011 \text { um } 20 \% \text { erhöhten Anteil am Export Usbekistans hatten, } \\
\text { Baumwolle hatte dagegen einen um } 1 \% \text { gesunkenen Anteil von } 12,7 \% \text {. }\end{array}$ \\
\hline 16.6.2012 & $\begin{array}{l}\text { In einer im ersten staatlichen Fernsehen übertragenen Rede vor örtlichen Deputieren im Gebiet Suchandarja } \\
\text { erklärt Präsident Karimow, dass das Heranwachsen einer gesunden jungen Generation und nicht die Steige- } \\
\text { rung der Bevölkerungszahl Ziel der Politik sei. }\end{array}$ \\
\hline 19.6 .2012 & $\begin{array}{l}\text { Olam.uz und UzDaily.uz melden unter Bezug auf den BP-Bericht zur globalen Energiesituation 2012, dass die } \\
\text { nachgewiesenen usbekischen Ölreserven mit } 100 \text { Mio. } t \text { und Gas 1,6 Bio. } \mathrm{m}^{3} \text { niedriger seien, als bisher vermutet. }\end{array}$ \\
\hline
\end{tabular}




\begin{tabular}{|l|l|}
\hline 19.6.2012 & $\begin{array}{l}\text { Im Gebiet Choresm werden drei Angehörige des medizinischen Personals unter dem Verdacht des Kinder- } \\
\text { handels verhaftet. Sie sollen in Zusammenarbeit mit einer Bande Neugeborene unverheirateter Mütter ver- } \\
\text { kauft haben. }\end{array}$ \\
\hline 19.6.2012 & $\begin{array}{l}\text { Der Sonderbeauftragte für Afghanistan und Pakistan des britischen Premierministers, Mark Sedwill, führt } \\
\text { während seines Taschkentbesuches Gespräche mit Außenminister Kamilow, Verteidigungsminister Berdi- } \\
\text { jew und Vertretern des Parlaments. }\end{array}$ \\
\hline 21.6.2012 & $\begin{array}{l}\text { Der Präsident der Moskauer Mutterfirma von MTS Usbekistan, Andrej Dubowskow, distanziert sich von } \\
\text { dem flüchtigen Generaldirektor Bekzod Achmedow, der illegale Geschäfte betrieben habe. }\end{array}$ \\
\hline 22.6.2012 & $\begin{array}{l}\text { Die beiden türkischen Unternehmer Mehmet Günes und Ali Kahwe werden von einem Gericht in Taschkent } \\
\text { wegen Steuerhinterziehung zu 11 und 24 Monaten Haft verurteilt, vier usbekische Mitarbeiter ihrer Firmen } \\
\text { Ilkai bzw. Pinar Trans müssen hohe Geldstrafen zahlen. }\end{array}$ \\
\hline
\end{tabular}

Sie können die gesamte Chronik seit 2008 auch auf http://www.laender-analysen.de/zentralasien/ unter dem Link »Chronik«lesen.

Die Zentralasien-Analysen werden vom Ost-Ausschuss der Deutschen Wirtschaft sowie zusätzlich von der GIZ unterstützt.

Ost-Ausschuss der Deutschen Wirtschaft
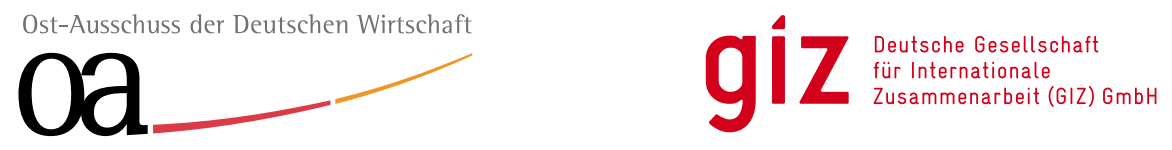

Herausgeber: Forschungsstelle Osteuropa an der Universität Bremen und Deutsche Gesellschaft für Osteuropakunde

Die Meinungen, die in den Zentralasien-Analysen geäußert werden, geben ausschließlich die Auffassung der Autoren wieder. Abdruck und sonstige publizistische Nutzung sind nach Rücksprache mit der Redaktion gestattet. Redaktion: Beate Eschment

Redaktionsassistenz: Alexander Wolters Satz: Matthias Neumann

Zentralasien-Analysen-Layout: Matthias Neumann, nach einen Konzept von Cengiz Kibaroglu, mit einer Grafik von Sebastian Klüsener Die Zentralasien-Analysen werden im Rahmen der Datenbank World Affairs Online (WAO) ausgewertet und sind im Portal IREON www.ireon-portal.de recherchierbar.

ISSN 1866-2110 @ 2012 by Deutsche Gesellschaft für Osteuropakunde e.V. und Forschungsstelle Osteuropa, Bremen

Deutsche Gesellschaft für Osteuropakunde e.V. • Schaperstraße 30 • 10719 Berlin • Telefon: +4930214784 12 • Telefax: +49 3021478414

e-mail:Zentralasien-Analysen@dgo-online.org•Internet-Adresse: www.laender-analysen.de/zentralasien 
Ost-Ausschuss der Deutschen Wirtschaft

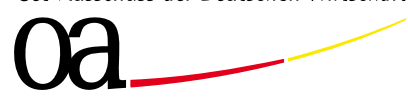

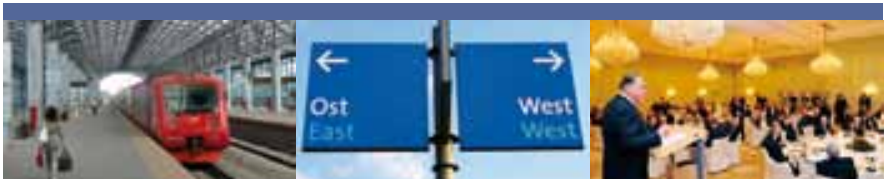

Der Ost-Ausschuss der Deutschen Wirtschaft arbeitet seit 1952 aktiv daran, die Handelsund Investitionsbedingungen für deutsche Unternehmen in über 20 Ländern des östlichen Europas und Zentralasiens zu verbessern.

Unsere Konferenzen und Fachseminare informieren Sie über aktuelle Entwicklungen zwischen Zagreb und Wladiwostok.
Wir sind die Stimme der deutschen Wirtschaft in bilateralen Gremien und vernetzen Wirtschaft und Politik durch die Organisation exklusiver Wirtschaftsgespräche und Delegationsreisen.

Mit unseren Projekten fördern wir die marktwirtschaftliche Entwicklung in Osteuropa und investieren in die Aus- und Weiterbildung junger Menschen.

Weitere Informationen zu unseren Veranstaltungen, Publikationen und zur Mitgliedschaft im Ost-Ausschuss finden Sie unter: www.ost-ausschuss.de

\title{
Brücke zu den Zukunftsmärkten
}

\author{
Ost-Ausschuss der Deutschen Wirtschaft
}




\title{
Kostenlose E-Mail-Dienste der Forschungsstelle Osteuropa und ihrer Partner auf www.laender-analysen.de
}

\author{
Belarus-Analysen \\ Die Belarus-Analysen bieten einmal alle zwei Monate eine Kurzanalyse zu einem aktuellen Thema aus Politik, Wirt- \\ schaft oder Kultur, ergänzt um Grafiken und Tabellen. Zusätzlich gibt es eine Chronik aktueller Ereignisse.
}

Abonnement unter: fsopr@uni-bremen.de

\section{Caucasus Analytical Digest}

Der Caucasus Analytical Digest bietet einmal monatlich englischsprachige Kurzanalysen sowie illustrierende Daten zu einem aktuellen Thema. Zusätzlich gibt es eine Chronik aktueller Ereignisse.

Abonnement unter: http://www.res.ethz.ch/analysis/cad/

\section{Polen-Analysen}

Die Polen-Analysen bieten zweimal monatlich eine Kurzanalyse zu einem aktuellen Thema aus Politik, Wirtschaft oder Kultur, ergänzt um Grafiken und Tabellen. Zusätzlich gibt es eine Chronik aktueller Ereignisse.

Abonnement unter: http://www.deutsches-polen-institut.de/Newsletter/subscribe.php

\section{Russland-Analysen}

Die Russlandanalysen bieten vierzehntägig eine Kurzanalyse zu einem aktuellen Thema, ergänzt um Grafiken und Tabellen. Zusätzlich gibt es eine Chronik aktueller Ereignisse.

Abonnement unter: fsopr@uni-bremen.de

\section{Russian Analytical Digest}

Der Russian Analytical Digest bietet zweimal monatlich englischsprachige Kurzanalysen sowie illustrierende Daten zu einem aktuellen Thema.

Abonnement unter: http://www.res.ethz.ch/analysis/rad/

\section{Ukraine-Analysen}

Die Ukraine-Analysen bieten zweimal monatlich eine Kurzanalyse zu einem aktuellen Thema aus Politik, Wirtschaft oder Kultur, ergänzt um Grafiken und Tabellen. Zusätzlich gibt es eine Chronik aktueller Ereignisse.

Abonnement unter: fsopr@uni-bremen.de

\section{Zentralasien-Analysen}

Die Zentralasien-Analysen bieten eine monatliche Kurzanalyse zu einem aktuellen Thema aus Politik, Wirtschaft oder Kultur, ergänzt um Grafiken und Tabellen. Zusätzlich gibt es eine Chronik aktueller Ereignisse.

Abonnement unter: Zentralasien-Analysen@dgo-online.org

\section{Bibliographische Dienste}

Die vierteljährlich erscheinenden Bibliographien informieren über englisch- und deutschsprachige Neuerscheinungen zu Belarus, Polen, Russland, Tschechischer und Slowakischer Republik, Ukraine sowie zu den zentralasiatischen und kaukasischen Staaten. Erfasst werden jeweils die Themenbereiche Politik, Außenpolitik, Wirtschaft und Soziales. 